\title{
Hamilton-Jacobi equations on networks as limits of singularly perturbed problems in optimal control: dimension reduction
}

\author{
Yves Achdou ${ }^{*}$ Nicoletta Tchou ${ }^{\dagger}$
}

March 19, 2014

\begin{abstract}
We consider a family of open star-shaped domains $\Omega^{\varepsilon}$ of $\mathbb{R}^{2}$. Roughly speaking, $\Omega^{\varepsilon}$ is made of a finite number of non intersecting semi-infinite strips of thickness $\varepsilon$ and of a central region whose diameter is of the order of $\varepsilon$, that may be called the junction. When the thickness $\varepsilon$ tends to 0 , the domains $\Omega^{\varepsilon}$ tend to a union of half-lines sharing an endpoint $O$. This set is termed network. We study infinite horizon optimal control problems in which the state is constrained to remain in $\overline{\Omega^{\varepsilon}}$. In the above mentioned strips the running cost may have a fast variation w.r.t. the transverse coordinate. We pass to the limit as the parameter $\varepsilon$ tends to zero, and prove that the value function tends to the solution of a Hamilton-Jacobi equation on the network, which may also be related to an optimal control problem. One difficulty is to find the transmission condition at the junction node $O$ in the limit problem. For passing to the limit, we use the method of the perturbed test-functions of Evans, which requires constructing suitable correctors. This is another difficulty since the domain is unbounded.
\end{abstract}

\section{Introduction}

A network (or a graph) is a set of items, referred to as vertices or nodes, with connections between them referred to as edges, see the right part of Figure 1 for an example. In the recent years there has been an increasing interest in the investigation of dynamical system and differential equation on networks, in particular in connection with problem of data transmission and traffic management (see for example Garavello-Piccoli [19], Engel et al [16]).

Nevertheless, the literature on continuous-state and continuous-time control on networks is still scarce: there is the recent article [1], where the authors (including those of the present paper) consider control problems whose dynamics is constrained to a network and related Bellman equations. They introduce a definition of viscosity solution which reduces to the usual one if the network is only composed of two parallel segments entering in a node: while in the interior of an edge one can test the equation with a smooth test-function, the main difficulties arise at the junctions where the network does not have a regular differential structure; at a vertex, a notion of derivative similar to that of Dini's derivative (see for example [9]) is proposed, hence admissible test-functions are the ones which admit derivatives in the directions of the edges sharing the node. With this definition, the intrinsic geodesic distance, fixed one argument, is an admissible test-function with respect to the other argument. The above mentioned notion

\footnotetext{
*Univ. Paris Diderot, Sorbonne Paris Cité, Laboratoire Jacques-Louis Lions, UMR 7598, UPMC, CNRS, F-75205 Paris, France. achdou@ljll.univ-paris-diderot.fr

${ }^{\dagger}$ IRMAR, Université de Rennes 1, Rennes, France, nicoletta.tchou@univ-rennes1.fr
} 
of viscosity solutions is equivalent to the one introduced independently by Imbert, Monneau and Zidani [21] for studying a Hamilton-Jacobi approach to junction problems and traffic flows. This was proved by Camilli and Marchi in [15]. There is also the work by Schieborn and Camilli [25], in which the authors focus on eikonal equations and on a less general notion of viscosity solution.

Both [1] and [21] contain the first comparison and uniqueness results, but in these works the assumptions are somewhat restrictive. More general comparison results have been proved in two very recent papers [2] and [20], which both handle more general transmission conditions at the crosspoints than in [1] and [21]. The paper by Imbert-Monneau [20] contains the most general comparison and uniqueness results: the proof, rather involved, is completely based on partial differential equations arguments and works for bimonotone Hamiltonians (not necessarily convex). In the more particular context of Hamilton-Jacobi equations coming from optimal control problems on networks, a short proof of a general comparison result is proposed in [2], whose authors use arguments from optimal control theory, adapting original ideas that Barles, Briani and Chasseigne, see [12, 11], have used for control problems with discontinuous dynamics and costs. In what follows, we shall use the comparison results of [20], which are summarized and adapted to the present context in $\S 4.2$ below.

The aim of this paper is to study the asymptotic behavior of the value function of an optimal control problem in which the dynamics is constrained to remain in the closure of a bidimensional thin open set $\Omega_{\varepsilon}$ converging to a network as the width parameter $\varepsilon$ tends to 0 . We restrict ourselves to the case of a single junction, i.e. the network has only one crosspoint: the set $\Omega_{\varepsilon}$ may be divided into a finite number of strips (which may be called the roads) and a junction zone, see the left part of Figure 1. Precise assumptions on the class of domains $\Omega_{\varepsilon}$ will be made in $\S 1.1$ below. In the roads (whose width is of the order of $\varepsilon$ ), the running cost may have fast variations in the transverse direction but not in the longitudinal direction. Similarly, in the roads, the state constraints involve only the (fast) transverse variable. This is why the problem may be termed a singularly perturbed problem.

In the case when there is no junction, singularly perturbed problems in optimal control have been studied by many authors, see for example Bensoussan [14], Artstein and Gaitsgory [7], Gaitsgory and Leizarowitz [18], Bagagiolo and Bardi [8], Alvarez and Bardi [3, 4], Terrone [26], Alvarez-Bardi-Marchi $[6,5]$. The reference [3] is of special interest here since the authors focus on Hamilton-Jacobi equations and use viscosity solutions arguments in order to study the asymptotic behavior of the value function: the method for proving the convergence to the dimension reduced effective problem is based on the perturbed test-function method of Evans [17], which implies the construction of correctors. The correctors are viscosity solution to some first order partial differential equation in the fast variable with state constrained boundary conditions.

The aim of the present paper is to perform the same kind of analysis for the family of the domains $\Omega_{\varepsilon}$ displayed on the left of Figure 1 as the width parameter $\varepsilon$ tends to 0 . The method uses viscosity solutions arguments and is reminiscent of that proposed in [3]. The effective equation far away from a junction is found in a straightforward manner by using the results contained in [3]. The main difficulty consists of finding the transmission conditions at the junction. Naturally, the latter depend on the dynamics and running cost in the junction zone. The convergence of the value function will be proved by using the comparison principle stated in [20]. The main technical point lies in the construction of junction-correctors and in their use in the perturbed test function method. The strategy for the construction of the junction-correctors is reminiscent of the one used by Ishii in [22]. An important difficulty comes from the unboundedness of the domain in which the correctors are defined: indeed, for obtaining bounded correctors, we will have to work in suitable unbounded subdomains obtained by truncating the original one. In 
turn, since the correctors are defined in subdomains, the method of the perturbed test functions of Evans will have to be suitably modified.

\section{$1.1 \quad$ The setting}

\subsubsection{The geometry}

For simplicity, let us focus on the model case of a star-shaped network with $N$ straight edges, $N>1$. Let $\left(e_{i}\right)_{i=1, \ldots, N}$ be a set of unit vectors in $\mathbb{R}^{2}$ s.t. $e_{j} \neq e_{k}$ if $j \neq k$. Note that $e_{j}=-e_{k}$ is possible. We assume that there is at least a pair $(j, k), j \neq k$ s.t. $e_{j}$ is not aligned with $e_{k}$. For any $i=1, \ldots, N$, let $e_{i}^{\perp}$ be a unit vector perpendicular to $e_{i}$. A given point $z \in \mathbb{R}^{2}$ is written $z=z_{i} e_{i}+z_{i}^{\perp} e_{i}^{\perp}$. The open half-line $\mathbb{R}_{+} e_{i}$ is denoted $G_{i}$, and the star shaped network $\mathcal{G}$ (see Figure 1 ) is defined by

$$
\mathcal{G}=\{O\} \cup \bigcup_{i=1}^{N} G_{i},
$$

where $O$ is the origin $O=(0,0)$.

For a radius $\rho>0$, let the convex polygonal domain $W_{\rho}$ be defined by

$$
W_{\rho}=\left\{z \in \mathbb{R}^{2}: \forall i=1, \ldots, N, z \cdot e_{i}<\rho\right\} .
$$

Let $\Omega$ be a connected open subset of $\mathbb{R}^{2}$ with the following properties, see Figure 1:

- $\Omega$ contains the origin $O$ and is star-shaped with respect to $O$

- $\Omega$ has a smooth boundary

- There exists a positive radius $r_{0}$ such that

$$
\Omega \backslash \bar{W}_{r_{0}}=\cup_{i=1}^{N} Z_{i},
$$

where $W_{\rho}$ is given by (1.1) and $Z_{i}$ is the half-strip

$$
Z_{i}=\left\{z=z_{i} e_{i}+z_{i}^{\perp} e_{i}^{\perp} ;-1<z_{i}^{\perp}<1 ; z_{i}>r_{0}\right\} .
$$

In other words, outside the region $\bar{W}_{r_{0}}, \Omega$ coincides with the union of the $N$ non intersecting semi-infinite strips of width 2 and aligned with the vectors $e_{i}, i=1, \ldots, N$.

Let $\widetilde{K}_{0}$ be the subset of $\Omega$ defined by

$$
\widetilde{K}_{0}=\Omega \cap W_{r_{0}}
$$

where $W_{\rho}$ is given by (1.1).

The control problems will take place in the set $\Omega_{\varepsilon}=\varepsilon \Omega$, which can be viewed as a thick version of $\mathcal{G}$. The thickness parameter $\varepsilon>0$ is bound to tend to zero.

\subsubsection{The control problem in $\Omega_{\varepsilon}$}

Let $A$ be a compact subset of $\mathbb{R}^{2}$ and $\ell_{\varepsilon}: \bar{\Omega}_{\varepsilon} \times A \rightarrow \mathbb{R}$ be a continuous function.

For a given positive number $\lambda$ and any $z_{0} \in \bar{\Omega}_{\varepsilon}$, we consider the infinite horizon control problem consisting of minimizing the cost functional

$$
J_{\varepsilon}\left(z_{0}, \alpha\right)=\int_{0}^{\infty} \ell_{\varepsilon}\left(z_{\varepsilon}\left(t ; z_{0}, \alpha\right), \alpha(t)\right) e^{-\lambda t} d t
$$




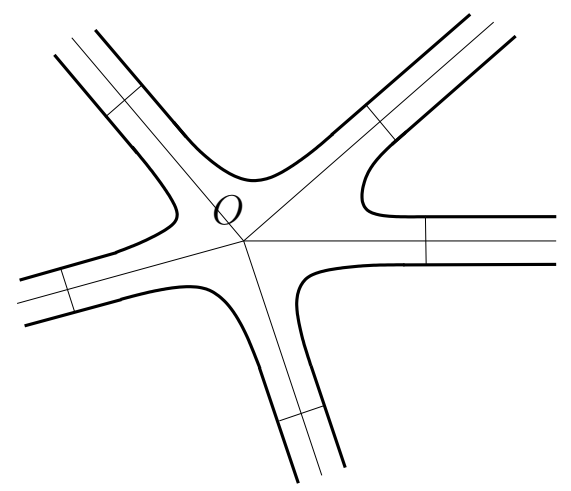

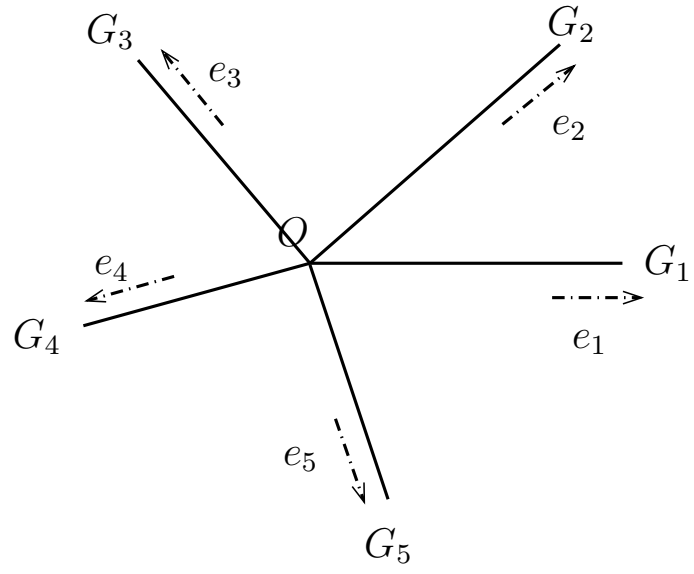

Figure 1: The set $\Omega$ and the network $\mathcal{G}(N=5)$

on the trajectories of the system

$$
\dot{z}_{\varepsilon}\left(t ; z_{0}, \alpha\right)=\alpha(t), \quad t>0, \quad z_{\varepsilon}\left(0 ; z_{0}, \alpha\right)=z_{0},
$$

where the control function $\alpha: \mathbb{R}_{+} \rightarrow A$ is measurable and such that the corresponding trajectory satisfies the state constraint $z_{\varepsilon}(t) \in \bar{\Omega}_{\varepsilon}$ for all $t \geq 0$. We start by making some assumptions on the structure of the problem.

Assumption 1.1. There exists a positive constant $r$ such that $A$ contains the ball $B(0, r)$.

Assumption 1.2. $\quad$ - The function $\ell_{\varepsilon}$ is continuous

- It has the following structure: there exist $N$ bounded and continuous functions $\ell_{i}:[0,+\infty) \times$ $[-1,1] \times A \rightarrow \mathbb{R}, i=1, \ldots, N$, and a function $\ell_{0}: \widetilde{K}_{0} \times A \rightarrow \mathbb{R}$ such that

$$
\begin{array}{ll}
\ell_{\varepsilon}(z, a)=\ell_{i}\left(z_{i}-\varepsilon r_{0}, \frac{z_{i}^{\perp}}{\varepsilon}, a\right) & \text { in } \varepsilon \bar{Z}_{i}, \\
\ell_{\varepsilon}(z, a)=\ell_{0}\left(\frac{z}{\varepsilon}, a\right) & \text { in } \varepsilon \widetilde{\widetilde{K}}_{0} .
\end{array}
$$

The functions $\ell_{i}$ are uniformly continuous in $[0,+\infty) \times[-1,1]$ uniformly w.r.t. $a \in A$. The function $\ell_{0}$ is uniformly continuous in ${\widetilde{\widetilde{K}_{0}}}_{0}$ uniformly w.r.t. $a \in A$.

Assumption 1.3. Since the function $\ell_{0}$ is bounded, it is not restrictive to assume that $\ell_{0}$ takes nonnegative values (just add a constant to $\ell_{0}$ if necessary).

Assumption 1.1 is on the controllability of the system. The most important part of Assumption 1.2 says that away from the junction, i.e. for $z$ belonging to the strip $\left[\varepsilon r_{0},+\infty\right) e_{i} \times[-\varepsilon, \varepsilon] e_{i}^{\perp}$, $1 \leq i \leq N$, the running cost has a fast dependence w.r.t. the transverse coordinate $z_{i}^{\perp}$ and a slow dependence w.r.t. the tangential coordinate $z_{i}$.

With Assumptions 1.1 and 1.2, it is well known, see e.g. [9] that the value function $u_{\varepsilon}$ of the control problem described above is bounded uniformly with respect to $\varepsilon$, continuous, and is the unique viscosity solution of

$$
\begin{array}{ll}
\lambda u_{\varepsilon}(z)+H_{\varepsilon}\left(z, D u_{\varepsilon}\right) \geq 0 & \text { in } \bar{\Omega}_{\varepsilon}, \\
\lambda u_{\varepsilon}(z)+H_{\varepsilon}\left(z, D u_{\varepsilon}\right) \leq 0 & \text { in } \Omega_{\varepsilon},
\end{array}
$$


where for $z \in \bar{\Omega}_{\varepsilon}, p \in \mathbb{R}^{2}$, the Hamiltonian $H_{\varepsilon}(z, p)$ is

$$
H_{\varepsilon}(z, p)=\max _{a \in A}\left(-p \cdot a-\ell_{\varepsilon}(z, a)\right) .
$$

Here $p \cdot a$ denotes the scalar product of $p$ by $a$.

\subsection{Organization of the paper}

Here, we propose an informal overview of the main result and of the notions that it requires. Since this paragraph is only meant to help the reader find her/his way in the paper, we do not mean to give full details, and rather refer to the places where the definitions are thoroughly written.

The main result is Theorem 4.2. Its statement is the following: under Assumptions 1.1-1.3 and the further Assumption 2.1, the sequence $u_{\varepsilon}$ converges locally uniformly to the bounded viscosity solution $u: \mathcal{G} \rightarrow \mathbb{R}$ of the Hamilton-Jacobi equation on $\mathcal{G}$,

$$
\left\{\begin{aligned}
\lambda u(x)+\bar{H}_{i}\left(x_{i}, \frac{d u}{d x_{i}}(x)\right) & =0 \quad x=x_{i} e_{i} \in G_{i}, \\
\lambda u(O)+\max \left(E, \bar{H}\left(O, \frac{d u}{d x_{1}}(0), \ldots, \frac{d u}{d x_{N}}(0)\right)\right) & =0
\end{aligned}\right.
$$

Let us list the necessary notions for this theorem and the places where they are defined:

1. The effective Hamiltonian $\bar{H}_{i}$ corresponding to the edge $G_{i}$ is defined in $\S 2$ :

$$
\bar{H}_{i}\left(x_{i}, p_{i}\right)=\sup _{\mu \in \mathcal{Z}_{i}}\left(\int_{[-1,1] \times A}\left(-p_{i} a_{i}-\ell_{i}\left(x_{i}, y, a\right)\right) d \mu(y, a)\right),
$$

where $\mathcal{Z}_{i}$ is a compact and convex set of Radon probability measures on $[-1,1] \times A$. This set may be viewed as a set of limiting relaxed controls. A technical assumption, useful for the construction of the junction-correctors, is introduced in $\S 2.3$.

2. The constant $E$ can be viewed as the opposite of an effective cost at the junction: it is defined in $\S 3$. It strongly depends on $\ell_{0}$.

3. The Hamiltonian $\bar{H}\left(O, \frac{d u}{d x_{1}}(0), \ldots, \frac{d u}{d x_{N}}(0)\right)$ appearing in the effective transmission condition at the junction is defined in $\S 4.1: \bar{H}(O, \cdot): \mathbb{R}^{N} \rightarrow \mathbb{R}$ is given by

$$
\bar{H}\left(O, p_{1}, \ldots, p_{N}\right) \equiv \max _{i=1, \ldots, N} \bar{H}_{i}^{+}\left(0, p_{i}\right),
$$

where

$$
\bar{H}_{i}^{+}\left(0, p_{i}\right)=\sup _{\mu \in \mathcal{Z}_{i}^{+}}\left(\int_{[-1,1] \times A}\left(-p_{i} a_{i}-\ell_{i}(0, y, a)\right) d \mu(y, a)\right),
$$

and

$$
\mathcal{Z}_{i}^{+}=\left\{\mu \in \mathcal{Z}_{i} \text { s.t. } \int_{[-1,1] \times A} a_{i} d \mu(y, a) \geq 0\right\} .
$$

The Hamiltonian $\bar{H}(O, \cdot)$ is thus constructed by considering only the controls for which the relaxed dynamics starting from $O$ point in one of the edges of $\mathcal{G}$ (or stay at $O$ ). 
4. The notion of viscosity solution of the above Hamilton-Jacobi equation has been defined in [1], and comparison results have been proved in [2] and [20]. These notions are reviewed in $\S 4.2$.

The main difficulties for proving Theorem 4.2 are the following:

- to identify the effective constant $E$ : in $\S 3.1, E$ is constructed as the limit of a sequence of ergodic constants related to larger and larger bounded subdomains of $\Omega$ with running $\operatorname{cost} \ell_{0}$.

- to construct correctors that will be used in the perturbed test-function argument of Evans, see [17]. The difficulty lies in the fact that the correctors need to be bounded functions defined in unbounded domains, ideally the whole domain $\Omega$. In general, it will not be possible to define these correctors in the full domain $\Omega$, and we will have to restrict ourselves to suitable unbounded subdomains of $\Omega$; The construction of correctors is done in $\S 5$. The proof of convergence is given in $\S 6$.

\section{The effective Hamiltonian in the edges}

The first step in understanding the asymptotic behavior of the value function $u_{\varepsilon}$ as $\varepsilon \rightarrow 0$ is to look at what happens far from the region $\varepsilon \widetilde{K}_{0}$, i.e. far from the junction. For that, it is possible to rely on existing results. In the whole Section $2, i$ is an index in $\{1, \ldots, N\}$.

\section{$2.1 \quad$ Known facts}

Singular perturbations in deterministic control have been studied by many authors from the viewpoint of either control theory or viscosity solutions. In particular, state constrained control problems in thin domains obtained by thickening a smooth manifold have been much studied.

The results of Alvarez-Bardi [3] and Terrone [26] are going to be used in the present particular setting.

For any $y_{0} \in[-1,1]$, let $\mathcal{A}_{i, y_{0}}$ be the set of measurable functions $\alpha: \mathbb{R}_{+} \rightarrow A$ such that the function $y: \mathbb{R}_{+} \rightarrow \mathbb{R}$ given by

$$
y(t) \equiv y_{0}+\int_{0}^{t} \alpha_{i}^{\perp}(s) d s
$$

satisfies the state constraint $y(s) \in[-1,1]$ for $s \in \mathbb{R}_{+}$. To summarize,

$$
\mathcal{A}_{i, y_{0}}=\left\{\alpha: \mathbb{R}_{+} \rightarrow A, \text { measurable, such that } y(s) \in[-1,1] \forall s \geq 0\right\},
$$

where $y(\cdot)$ is given by $(2.1)$.

It is possible to define the effective Hamiltonians relative to the edges: let $H_{i}:[0,+\infty] \times[-1,1] \times$ $\mathbb{R}^{2} \rightarrow \mathbb{R}$ be defined by

$$
H_{i}(x, y, p)=\max _{a \in A}\left(-p \cdot a-\ell_{i}(x, y, a)\right) .
$$

Theorem 2.1 (Alvarez-Bardi [3]). For any number $x_{i} \geq 0$ and for any $p_{i} \in \mathbb{R}$, there exists a unique real number $\bar{H}_{i}\left(x_{i}, p_{i}\right)$ such that the problem

$$
\begin{array}{ll}
H_{i}\left(x_{i}, y, p_{i} e_{i}+D_{y} \chi_{i}(y) e_{i}^{\perp}\right) \geq \bar{H}_{i}\left(x_{i}, p_{i}\right) & y \in[-1,1], \\
H_{i}\left(x_{i}, y, p_{i} e_{i}+D_{y} \chi_{i}(y)\right) e_{i}^{\perp} \leq \bar{H}_{i}\left(x_{i}, p_{i}\right) & y \in(-1,1)
\end{array}
$$


has a Lipschitz continuous viscosity solution $\chi_{i}:[-1,1] \rightarrow \mathbb{R}$.

Moreover, for any $y_{0} \in[-1,1]$,

$$
\bar{H}_{i}\left(x_{i}, p_{i}\right)=\lim _{\rho \rightarrow 0+} \rho w_{\rho, x_{i}, p_{i}}\left(y_{0}\right),
$$

where

$$
w_{\rho, x_{i}, p_{i}}\left(y_{0}\right)=\sup _{\mathcal{A}_{i, y_{0}}} \int_{0}^{\infty} e^{-\rho s}\left(-p_{i} \alpha_{i}(s)-\ell_{i}\left(x_{i}, y(s), \alpha(s)\right)\right) d s,
$$

and $\mathcal{A}_{i, y_{0}}$ and $y$ are defined in (2.2) and (2.1). The limit in (2.5) is uniform w.r.t. $y_{0}$.

In [3], it is proved that for $y(s)$ and $\mathcal{A}_{i, y_{0}}$ as in $(2.2), \bar{H}_{i}\left(x_{i}, p_{i}\right)$ may be characterized with long time limits:

$$
\bar{H}_{i}\left(x_{i}, p_{i}\right)=\sup _{\mathcal{A}_{i, y_{0}}} \limsup _{t \rightarrow \infty} \frac{1}{t} \int_{0}^{t}\left(-p_{i} \alpha_{i}(s)-\ell_{i}\left(x_{i}, y(s), \alpha(s)\right)\right) d s,
$$

for all $y_{0} \in[-1,1]$.

In the same article [3], the following characterization of $\bar{H}_{i}\left(x_{i}, p_{i}\right)$ is given, as a rescaled limit of the value function of a finite horizon problem:

$$
\bar{H}_{i}\left(x_{i}, p_{i}\right)=\lim _{t \rightarrow \infty} \sup _{\mathcal{A}_{i, y_{0}}} \frac{1}{t} \int_{0}^{t}\left(-p_{i} \alpha_{i}(s)-\ell_{i}\left(x_{i}, y(s), \alpha(s)\right)\right) d s,
$$

for all $y_{0} \in[-1,1]$. For the latter, Alvarez and Bardi consider the value function

$$
v_{i}\left(x_{i}, p_{i}, y_{0}, t\right)=\inf _{\alpha \in \mathcal{A}_{i, y_{0}}}\left\{\int_{0}^{t}\left(p_{i} \alpha_{i}(s)+\ell_{i}\left(x_{i}, y(s), \alpha(s)\right)\right) d s\right\}
$$

and prove that there exists a constant $C$ independent of $y_{0}$ and $t$ (but which may depend on $x_{i}$ and $p_{i}$ ) such that

$$
\left|v_{i}\left(x_{i}, p_{i}, y_{0}, t\right)+t \bar{H}_{i}\left(x_{i}, p_{i}\right)\right| \leq C .
$$

Limiting relaxed controls and limit control problem It is possible to construct an optimal control problem whose Hamiltonian is $\bar{H}_{i}$.

Let $([-1,1] \times A)^{r}$ be the set of Radon probability measures on $[-1,1] \times A$. For a function $f: \mathbb{R} \times[-1,1] \times A \rightarrow \mathbb{R}$, let $f^{r}$ be defined by $f^{r}(x, \mu)=\int_{[-1,1] \times A} f(x, y, a) d \mu(y, a)$, for all $x \in \mathbb{R}$ and $\mu \in([-1,1] \times A)^{r}$.

A sequence of Radon probability measures $\mu_{n}$ on $[-1,1] \times A$ is said to converge weak-* to $\mu$ if for any continuous function $\psi$ on $[-1,1] \times A, \lim _{n \rightarrow \infty} \int_{[-1,1] \times A} \psi(y, a) d \mu_{n}(y, a)=\int_{[-1,1] \times A} \psi(y, a) d \mu(y, a)$. The Prokhorov distance $\pi(\cdot, \cdot)$ is defined for any pair $\left(\mu_{1}, \mu_{2}\right)$ of probability measures on $[-1,1] \times$ $A$ by

$$
\pi\left(\mu_{1}, \mu_{2}\right)=\inf \left\{\varepsilon>0: \mu_{1}(Q) \leq \mu_{2}(Q+\varepsilon B)+\varepsilon \text { for any measurable } Q\right\},
$$

where $B$ is the unit ball in $\mathbb{R}^{3}$. It is well known that this distance has the following property: any sequence $\mu_{n}$ of Radon probability measures on $[-1,1] \times A$ converges weak-* to $\mu$ if and only if $\lim _{n \rightarrow \infty} \pi\left(\mu_{n}, \mu\right)=0$. The set $([-1,1] \times A)^{r}$ equipped with the distance $\pi$ is a compact metric space.

If $\mathcal{P}$ is a subset of $([-1,1] \times A)^{r}$ and $\mu$ is an element of $([-1,1] \times A)^{r}$, then we define the distance 
$\pi(\mu, \mathcal{P})$ as the infimum of $\{\pi(\mu, \nu), \nu \in \mathcal{P}\}$. For two subsets $\mathcal{P}_{1}$ and $\mathcal{P}_{2}$ of $([-1,1] \times A)^{r}$, the Hausdorff distance $\pi_{H}\left(\mathcal{P}_{1}, \mathcal{P}_{2}\right)$ is defined as

$$
\pi_{H}\left(\mathcal{P}_{1}, \mathcal{P}_{2}\right)=\max \left(\sup _{\mu \in \mathcal{P}_{1}} \pi\left(\mu, \mathcal{P}_{2}\right), \sup _{\mu \in \mathcal{P}_{2}} \pi\left(\mu, \mathcal{P}_{1}\right)\right) .
$$

For any control law $\alpha \in \mathcal{A}_{i, y_{0}}$, let $y(t)$ be given by (2.1). For $s>0$, the occupational measure $\mu_{s}$ generated by $(y(t), \alpha(t))$ is the Radon probability measure defined on the Borel $\sigma$-algebra of $\mathbb{R} \times A$ by

$$
\mu_{s}=\frac{1}{s} \int_{0}^{s} \delta_{(y(t), \alpha(t))} d t
$$

where $\delta_{(y(t), \alpha(t))}$ is the Dirac mass concentrated at $(y(t), \alpha(t))$.

For $s>0$, let $\mathcal{Z}\left(s ; i, y_{0}\right)$ be the set of the occupational measures generated by the trajectories $(y(t), \alpha(t))$ up to time $s$ where $\alpha$ belongs to $\mathcal{A}_{i, y_{0}}$ and $y(s)$ is given by (2.1). It has been proved in $[18]$ that there exists a subset $\mathcal{Z}\left(i, y_{0}\right)$ of $([-1,1] \times A)^{r}$ such that

$$
\lim _{s \rightarrow \infty} \pi_{H}\left(\mathcal{Z}\left(s ; i, y_{0}\right), \mathcal{Z}\left(i, y_{0}\right)\right)=0 .
$$

The set $\mathcal{Z}\left(i, y_{0}\right)$ is convex and compact in the weak-* topology. Moreover, under Assumption 1.1 , the set $\mathcal{Z}\left(i, y_{0}\right)$ does not depend on $y_{0} \in[-1,1]$. This set is called the limit occupational measure set and will be noted $\mathcal{Z}_{i}$.

It has been proved by Terrone[26] that $\mathcal{Z}_{i}$ coincides with the set of limiting relaxed controls, i.e. the set of the probability Radon measures $\mu$ on $[-1,1] \times A$ such that there exists a control law $\alpha \in \mathcal{A}_{i, y_{0}}$ and a sequence $t_{n} \rightarrow+\infty$ such that the corresponding sequence of occupational measures $\mu_{t_{n}}$ generated by (2.1) converges to $\mu$ weak-*.

Then using the results in [3], we see that

$$
\mathcal{Z}_{i} \subset\left\{\mu \in([-1,1] \times A)^{r}, \int_{[-1,1] \times A} a_{i}^{\perp} d \mu(y, a)=0\right\} .
$$

It is proved in [3] that

$$
\bar{H}_{i}\left(x_{i}, p_{i}\right)=\sup _{\mu \in \mathcal{Z}_{i}}\left(-p_{i} \int_{[-1,1] \times A} a_{i} d \mu(y, a)-\ell_{i}^{r}\left(x_{i}, \mu\right)\right) .
$$

It is clear that $\bar{H}_{i}$ is continuous, convex with respect to the second variable, and that for all $x_{i}$, $\lim _{p_{i} \rightarrow \infty} \bar{H}_{i}\left(x_{i}, p_{i}\right)=+\infty$. The infinite horizon control problem associated with the Hamiltonian $\bar{H}_{i}$ is the minimization of

$$
\bar{J}_{i}(x, \mu)=\int_{0}^{\infty} e^{-\lambda t} \ell_{i}^{r}\left(x(t), \mu_{t}\right) d t
$$

for the system

$$
\dot{x}(t)=\int_{[-1,1] \times A} a_{i} d \mu_{t}(y, a), \quad \mu_{t} \in \mathcal{Z}_{i}, \quad x(0)=x .
$$

The affine-convex case It is proved in [3] that if $A$ is convex and $\ell_{i}$ is convex with respect to its last two variables $\left(y_{i}^{\perp}, a\right)$, then $\bar{H}_{i}\left(x_{i}, p_{i}\right)$ is characterized by

$$
\bar{H}_{i}\left(x_{i}, p_{i}\right)=\max _{a \in A, a_{i}^{+}=0, y \in[-1,1]}\left(-p_{i} a_{i}-\ell_{i}\left(x_{i}, y, a\right)\right) .
$$


If furthermore, $\ell_{i}$ does not depend on $y$, then

$$
\bar{H}_{i}\left(x_{i}, p_{i}\right)=\max _{a \in A, a_{i}^{\perp}=0}\left(-p_{i} a_{i}-\ell_{i}\left(x_{i}, a\right)\right) .
$$

The case of an asymptotically stable optimal trajectory The characterization of $\bar{H}_{i}\left(x_{i}, p_{i}\right)$ by (2.11) can be obtained in other situations than the affine-convex case: here, following Example 7.4 in [7], we assume that for all $x_{i}, p_{i}$, there exists some $y_{0} \in[-1,1]$ and some control $\alpha^{*} \in \mathcal{A}_{i, y_{0}}$ such that

$$
\int_{0}^{t}\left(p_{i} \alpha_{i}^{*}(s)+\ell_{i}\left(x_{i}, y^{*}(s), \alpha^{*}(s)\right)\right) d s=v_{i}\left(x_{i}, p_{i}, y_{0}, t\right), \quad \forall t>0,
$$

where $\dot{y}^{*}(s)=\alpha_{i}^{*, \perp}(s)$ and $y^{*}(0)=y_{0}$, and that

$$
\lim _{t \rightarrow \infty} \alpha^{*}(t)=a^{*} \text { and } \lim _{t \rightarrow \infty} y^{*}(t)=y^{*} .
$$

Then $a_{i}^{*, \perp}=0$ and $\lim _{t \rightarrow \infty} \frac{1}{t} \int_{0}^{t}\left(p_{i} \alpha_{i}^{*}(s)+\ell_{i}\left(x_{i}, y^{*}(s), \alpha^{*}(s)\right)\right) d s=p_{i} a_{i}^{*}+\ell_{i}\left(x_{i}, y^{*}, a^{*}\right)$. This implies (2.11), see [3].

\subsection{The minimal value of $\bar{H}_{i}(0, \cdot)$}

Define $\Lambda_{i}^{0}$ by

$$
\Lambda_{i}^{0}=\min _{p \in \mathbb{R}} \bar{H}_{i}(0, p) .
$$

Since $\lim _{|p| \rightarrow \infty} \bar{H}_{i}(0, p)=+\infty$ and $\bar{H}_{i}(0, \cdot)$ is convex, the set $\operatorname{argmin} H_{i}(0, \cdot)$ is a nonempty bounded interval, see Figure 4 for an example: let $\underline{p}_{i} \geq \bar{p}_{i}$ be the endpoints of $\operatorname{argmin} H_{i}(0, \cdot)$ :

$$
\operatorname{argmin} H_{i}(0, \cdot)=\left\{p \in \mathbb{R}, \bar{H}_{i}(0, p)=\Lambda_{i}^{0}\right\}=\left[\underline{p}_{i}, \bar{p}_{i}\right] .
$$

Let us denote by $\mathcal{Z}_{i}^{0}$ and $\mathcal{Z}_{i}^{+}$the following convex and compact subsets of $\mathcal{Z}_{i}$ :

$$
\begin{aligned}
& \mathcal{Z}_{i}^{0}=\left\{\mu \in \mathcal{Z}_{i} \text { s.t. } \int_{[-1,1] \times A} a_{i} d \mu(y, a)=0\right\}, \\
& \mathcal{Z}_{i}^{+}=\left\{\mu \in \mathcal{Z}_{i} \text { s.t. } \int_{[-1,1] \times A} a_{i} d \mu(y, a) \geq 0\right\} .
\end{aligned}
$$

These sets are non empty from Assumption 1.1.

Lemma 2.1. $\quad$ 1. $p_{i}^{0} \in \operatorname{argmin} H_{i}(0, \cdot)$ if and only if there exists $\mu^{*} \in \mathcal{Z}_{i}^{0}$ such that

$$
\bar{H}_{i}\left(0, p_{i}^{0}\right)=-\ell_{i}^{r}\left(0, \mu^{*}\right)
$$

2.

$$
\Lambda_{i}^{0}=-\min _{\mu \in \mathcal{Z}_{i}^{0}} \ell_{i}^{r}(0, \mu)
$$

3. If $p \geq \underline{p}_{i}$, then

$$
\max _{\mu \in \mathcal{Z}_{i}^{+}} \int_{[-1,1] \times A}\left(-p a_{i}-\ell_{i}(0, y, a)\right) d \mu(y, a)=\Lambda_{i}^{0} .
$$


Proof. The Hamiltonian $\bar{H}_{i}(0, \cdot)$ reaches its minimum at $p_{i}^{0}$ if and only if $0 \in \partial \bar{H}_{i}\left(0, p_{i}^{0}\right)$. The subdifferential of $\bar{H}_{i}(0, \cdot)$ at $p_{i}^{0}$ is characterized by

$\partial \bar{H}_{i}\left(0, p_{i}^{0}\right)=\overline{\mathrm{co}}\left\{-\int_{[-1,1] \times A} a_{i} d \mu(y, a) ; \mu \in \mathcal{Z}_{i}\right.$ s.t. $\left.\bar{H}_{i}\left(0, p_{i}^{0}\right)=-p_{i}^{0} \int_{[-1,1] \times A} a_{i} d \mu(y, a)-\ell_{i}^{r}(0, \mu)\right\}$,

see [27]. But the set

$$
\left\{\mu \in \mathcal{Z}_{i} \text { s.t. } \bar{H}_{i}\left(0, p_{i}^{0}\right)=-p_{i}^{0} \int_{[-1,1] \times A} a_{i} d \mu(y, a)-\ell_{i}^{r}(0, \mu)\right\}
$$

is compact and convex. Hence,

$\partial \bar{H}_{i}\left(0, p_{i}^{0}\right)=\left\{-\int_{[-1,1] \times A} a_{i} d \mu(y, a) ; \mu \in \mathcal{Z}_{i}\right.$ s.t. $\left.\bar{H}_{i}\left(0, p_{i}^{0}\right)=-p_{i}^{0} \int_{[-1,1] \times A} a_{i} d \mu(y, a)-\ell_{i}^{r}(0, \mu)\right\}$.

Therefore, $0 \in \partial \bar{H}_{i}\left(0, p_{i}^{0}\right)$ if and only if there exists $\mu^{*} \in \mathcal{Z}_{i}$ such that $\int_{[-1,1] \times A} a_{i} d \mu^{*}(y, a)=0$ and $\bar{H}_{i}\left(0, p_{i}^{0}\right)=-\ell_{i}^{r}\left(0, \mu^{*}\right)$. We have proved point 1 .

Point 2 is a direct consequence of point 1 .

If $p \geq \underline{p}_{i}$, then

$\max _{\mu \in \mathcal{Z}_{i}^{+}}\left(-p \int_{[-1,1] \times A} a_{i} d \mu(y, a)-\ell_{i}^{r}(0, \mu)\right) \leq \max _{\mu \in \mathcal{Z}_{i}^{+}}\left(-\underline{p}_{i} \int_{[-1,1] \times A} a_{i} d \mu(y, a)-\ell_{i}^{r}(0, \mu)\right)=\bar{H}_{i}\left(0, \underline{p}_{i}\right)$

where the last identity comes from point 1.

On the other hand,

$$
\max _{\mu \in \mathcal{Z}_{i}^{+}}\left(-p \int_{[-1,1] \times A} a_{i} d \mu(y, a)-\ell_{i}^{r}(0, \mu)\right) \geq-\min _{\mu \in \mathcal{Z}_{i}^{0}} \ell_{i}^{r}(0, \mu) .
$$

Point 3 is obtained by combining the two previous observations and point 2 .

Remark 2.1. From Assumption 1.3 and Lemma 2.1, we see that $\Lambda_{i}^{0}$ is non positive.

\subsection{A further assumption on $\ell_{i}(0, \cdot, \cdot)$}

Assumption 2.1 below will turn useful for studying the asymptotics of $u_{\varepsilon}$ near the junction; roughly speaking, it says that for any real number $p_{i}<\underline{p}_{i}$ (which implies $\bar{H}_{i}\left(0, p_{i}\right)>\Lambda_{i}^{0}$ ), for all $t$, it is possible to construct an admissible control law $\tilde{\alpha}$ whose related cost remains at a given distance of the optimal value $v_{i}\left(0, p_{i}, y_{0}, t\right)$, and for which the $i$ coordinate, namely $s \in[0, t] \mapsto \int_{0}^{s} \tilde{\alpha}_{i}(\theta) d \theta$ remains bounded from below by a fixed constant. Assumption 2.1 will be used in $\S 5$ for constructing the correctors near the junction.

Proposition 2.1 and 2.2 below supply examples when Assumption 2.1 holds.

Assumption 2.1. 1. For any real number $p_{i}$ such that $p_{i}<p_{i}$, there exist two constants $L_{i} \geq 0$ and $C_{i}>0$ such that for all $y_{0} \in[-1,1]$, for all $t>0$, there exists a control law $\tilde{\alpha} \in \mathcal{A}_{i, y_{0}}$ such that

$$
\begin{array}{r}
\int_{0}^{s} \tilde{\alpha}_{i}(\tau) d \tau \geq-L_{i}, \quad \forall 0 \leq s \leq t, \\
\int_{0}^{t}\left(p_{i} \tilde{\alpha}_{i}(s)+\ell_{i}(0, \tilde{y}(s), \tilde{\alpha}(s))\right) d s \leq v_{i}\left(0, p_{i}, y_{0}, t\right)+C_{i},
\end{array}
$$


where $\tilde{y}(t)=y_{0}+\int_{0}^{t} \tilde{\alpha}_{i}^{\perp}(s) d s$.

2. For $p_{i}$ such that $p_{i} \leq p_{i} \leq \bar{p}_{i}$, (hence $\left.\bar{H}_{i}\left(0, p_{i}\right)=\Lambda_{i}^{0}\right)$, there exist two constants $L_{i} \geq 0$ and $C_{i}>0$ such that for all $y_{0} \in[-1,1]$, for all $t>0$, there exists a control law $\tilde{\alpha} \in \mathcal{A}_{i, y_{0}}$ such that

$$
\begin{array}{r}
-L_{i} \leq \int_{0}^{s} \tilde{\alpha}_{i}(\tau) d \tau \leq L_{i}, \quad \forall 0 \leq s \leq t, \\
\int_{0}^{t} \ell_{i}(0, \tilde{y}(s), \tilde{\alpha}(s)) d s \leq v_{i}\left(0, p_{i}, y_{0}, t\right)+C_{i},
\end{array}
$$

where $\tilde{y}(t)=y_{0}+\int_{0}^{t} \tilde{\alpha}_{i}^{\perp}(s) d s$.

Remark 2.2. Note that from (2.9), (2.19) is equivalent to

$$
\int_{0}^{t}\left(p_{i} \tilde{\alpha}_{i}(s)+\ell_{i}(0, \tilde{y}(s), \tilde{\alpha}(s))\right) d s \leq-t \bar{H}_{i}\left(0, p_{i}\right)+C_{i, 2},
$$

for a constant $C_{i, 2}$, and that (2.20)(2.21) imply (2.22).

Proposition 2.1. Under Assumptions 1.1, 1.2 and 1.3, if the set $A$ is convex and the function $\ell_{i}(0, \cdot, \cdot)$ is convex on $[-1,1] \times A$, then Assumption 2.1 holds.

Proof. The proof is given in the appendix.

Remark 2.3. The conclusion of Proposition 2.1 holds when there is an asymptotically stable optimal trajectory, see the paragraph at the end of $\S 2.1$.

Proposition 2.2. Under Assumptions 1.1, 1.2 and 1.3, if there exist two positive constants $\underline{c}_{i} \leq \bar{c}_{i}$ and an exponent $\nu_{i}>1$ such that for all $y \in[-1,1]$ and $a \in A$,

$$
-\Lambda_{i}^{0}+\underline{c}_{i}|a|^{\nu_{i}} \leq \ell_{i}(0, y, a) \leq-\Lambda_{i}^{0}+\bar{c}_{i}|a|^{\nu_{i}}
$$

where $\Lambda_{i}^{0}$ is defined in (2.13), then Assumption 2.1 holds.

Proof. The proof is given in the appendix.

\section{A scalar quantity that will appear in the effective equation at the junction}

In what follows, we will make a blow-up near the junction point $O$ : this leads us to extend the function $\ell_{0}$ to the whole domain $\bar{\Omega}$ by setting for any $a \in A$

$$
\ell_{0}(z, a)=\ell_{i}\left(0, z_{i}^{\perp}, a\right), \quad \text { if } z_{i} \geq r_{0},\left|z_{i}^{\perp}\right| \leq 1 .
$$

\subsection{Ergodic constants for state constraint problems in bounded subdomains}

For any real number $R$ such that $R>r_{0}$, let the bounded and connected open set $\Omega^{R}$ be defined by

$$
\Omega^{R}=\Omega \cap W_{R},
$$

where $W_{R}$ is given by (1.1). Consider the infinite horizon control problem

$$
w_{\rho}^{R}\left(z_{0}\right)=\inf _{\mathcal{A}_{z_{0}}^{R}} \int_{0}^{\infty} e^{-\rho s} \ell_{0}(z(s), \alpha(s)) d s,
$$


where

$$
\mathcal{A}_{z_{0}}^{R}=\left\{\alpha: \mathbb{R}_{+} \rightarrow A, \text { measurable, such that } z(s) \equiv z_{0}+\int_{0}^{s} \alpha(\theta) d \theta \in \bar{\Omega}^{R}, \forall s \geq 0\right\} .
$$

It is easy to prove that $w_{\rho}^{R}$ is the unique viscosity solution of the following problem:

$$
\begin{array}{ll}
\rho w_{\rho}^{R}+H_{0}\left(\cdot, D w_{\rho}^{R}\right) \geq 0 & \text { in } \bar{\Omega}^{R}, \\
\rho w_{\rho}^{R}+H_{0}\left(\cdot, D w_{\rho}^{R}\right) \leq 0 & \text { in } \Omega^{R},
\end{array}
$$

where for $z \in \bar{\Omega}^{R}, p \in \mathbb{R}^{2}$, the Hamiltonian $H_{0}(z, p)$ is

$$
H_{0}(z, p)=\max _{a \in A}\left(-p \cdot a-\ell_{0}(z, a)\right) .
$$

Using the hypotheses on controllability, continuity and boundedness of the data, it is now standard (see [24]) to obtain the existence of a unique ergodic constant $E^{R}$ and of a bounded Lipschitz corrector $w^{R}$ such that

$$
\begin{array}{ll}
H_{0}\left(\cdot, D w^{R}\right) \geq E^{R} & \text { in } \bar{\Omega}^{R}, \\
H_{0}\left(\cdot, D w^{R}\right) \leq E^{R} & \text { in } \Omega^{R},
\end{array}
$$

where (up to a subsequence) $w^{R}(z)=\lim _{\rho \rightarrow 0} w_{\rho}^{R}(z)-<w_{\rho}^{R}>$ and $E^{R}=-\lim _{\rho \rightarrow 0} \rho w_{\rho}^{R}(z)$.

The ergodic constant $E^{R}$ is bounded uniformly w.r.t. $R$ and there exists a constant $C_{L}$ independent of $R$ such that $\left|D w^{R}(z)\right| \leq C_{L}$. Furthermore, if $R \leq R^{\prime}$, the inclusion $\mathcal{A}_{z}^{R} \subset \mathcal{A}_{z}^{R^{\prime}}$ yields that $w_{\rho}^{R}(z) \geq w_{\rho}^{R^{\prime}}(z)$. This implies that if $R \leq R^{\prime}$, then $E^{R} \leq E^{R^{\prime}}$.

\subsection{Passing to the limit as $R \rightarrow+\infty$ : the constant $E$}

The boundedness and the monotony of the application $R \mapsto E^{R}$ make it possible to pass to the limit and define a limit ergodic constant

$$
E \equiv \lim _{R \rightarrow \infty} E^{R}
$$

The constant $E$ will play an important role in the effective equation at the junction.

Example 3.1. It is easy to see that if there exists $z_{0} \in \tilde{K}_{0}$ such that $\ell_{0}\left(z_{0}, 0\right)=0$, then $E=0$.

Remark 3.1. In what follows, we shall use the fact that, for any $\varepsilon>0$, there exists $R(\varepsilon)>0$ such that $\forall r>R(\varepsilon), w^{r}$ is a viscosity supersolution of

$$
H_{0}\left(\cdot, D w^{r}\right) \geq E-\varepsilon, \quad \text { in } \bar{\Omega}^{r} .
$$

Remark 3.2. Using the uniform coercivity of $H_{0}$, there exists $q$ such that $H_{0}(z, p) \geq E+2$ for any $z \in \bar{\Omega}, p \in \mathbb{R}^{2}$ with $|p| \geq q$.

Let $R$ be any fixed real number such that $R>r_{0}$. For any $M \geq q$, consider the continuous extension $\tilde{w}^{R}$ of $w^{R}$, defined for $z=z_{i} e_{i}+z_{i}^{\perp} e_{i}^{\perp}, z_{i} \in(R,+\infty), z_{i}^{\perp} \in[-1,1]$ by

$$
\tilde{w}^{R}(z):=w^{R}\left(z^{R}\right)+M\left(z-z^{R}\right), \quad \text { with } z^{R}=R e_{i}+z_{i}^{\perp} e_{i}^{\perp} .
$$

It is clear that $\tilde{w}^{R}$ is a continuous extension of $w^{R}$ to $\bar{\Omega}$.

Moreover, for $R>R(\varepsilon)$ as in Remark 3.1, it is easy to see that $\tilde{w}^{R}$ is a viscosity supersolution of

$$
H_{0}\left(\cdot, D \tilde{w}^{R}\right) \geq E-\varepsilon \quad \text { in } \bar{\Omega} .
$$




\subsection{Estimates for some trajectories which start in $\widetilde{K}_{0}$ and return there after some time}

For $z_{0} \in \bar{\Omega}$, let $\mathcal{A}_{z_{0}}$ be the set

$$
\mathcal{A}_{z_{0}}=\left\{\alpha: \mathbb{R}_{+} \rightarrow A, \text { measurable, such that } \forall s \geq 0, z(s) \equiv z_{0}+\int_{0}^{s} \alpha(\theta) d \theta \in \bar{\Omega}\right\} .
$$

Lemma 3.1. There exists a constant $C$ such that for all $z_{0} \in \widetilde{K}_{0}$, (see (1.3) for the definition of $\left.\widetilde{K}_{0}\right)$, for all $\alpha \in \mathcal{A}_{z_{0}}$ and $T_{0}>0$ such that $z\left(T_{0}\right) \in \widetilde{K}_{0}$, (recall that $z(s) \equiv z_{0}+\int_{0}^{s} \alpha(\theta)$ ),

$$
\int_{0}^{T_{0}} \ell_{0}(z(s), \alpha(s)) d s \geq-E T_{0}-C .
$$

Proof. There exists a time $\tau \geq 0$ (which can be bounded by a constant depending only on the controllability and bounds in our hypotheses (on $\ell$ and $A$ )) and a control law $\tilde{\alpha}(\cdot)$ such that $z\left(T_{0}\right)+\int_{0}^{t} \tilde{\alpha}(s) d s \in \widetilde{K}_{0}$ for any $t \in[0, \tau]$ and $z\left(T_{0}\right)+\int_{0}^{\tau} \tilde{\alpha}(s) d s=z_{0}$. The following control law

$$
\alpha^{*}(t)= \begin{cases}\alpha(t) & \text { if } t \in\left[0, T_{0}\right], \\ \tilde{\alpha}\left(t-T_{0}\right) & \text { if } t \in\left(T_{0}, T_{0}+\tau\right],\end{cases}
$$

can be extended by periodicity to $\mathbb{R}^{+}$(the period is $T_{0}+\tau$ ) to yield an admissible periodic trajectory $z^{*}(t) \equiv z_{0}+\int_{0}^{t} \alpha^{*}(s) d s$. Then,

$$
\int_{0}^{\infty} e^{-\rho s} \ell_{0}\left(z^{*}(s), \alpha^{*}(s)\right) d s=\sum_{k=0}^{\infty} \int_{k\left(T_{0}+\tau\right)}^{(k+1)\left(T_{0}+\tau\right)} e^{-\rho s} \ell_{0}\left(z^{*}(s), \alpha^{*}(s)\right) d s,
$$

and

$$
\sum_{k=0}^{\infty} e^{-k \rho\left(T_{0}+\tau\right)}\left(\int_{0}^{T_{0}+\tau} e^{-\rho s} \ell_{0}\left(z^{*}(s), \alpha^{*}(s)\right) d s\right)=\frac{1}{1-e^{-\rho\left(T_{0}+\tau\right)}} \int_{0}^{T_{0}+\tau} e^{-\rho s} \ell_{0}\left(z^{*}(s), \alpha^{*}(s)\right) d s .
$$

This implies that

$$
\lim _{\rho \rightarrow 0} \rho \int_{0}^{\infty} e^{-\rho s} \ell_{0}\left(z^{*}(s), \alpha^{*}(s)\right) d s=\frac{1}{T_{0}+\tau} \int_{0}^{T_{0}+\tau} \ell_{0}\left(z^{*}(s), \alpha^{*}(s)\right) d s .
$$

Moreover, it is possible to choose $R$ large enough such that $z^{*}(t) \in \Omega^{R}$ for all $t \in\left[0, T_{0}+\tau\right]$. Therefore, up to a subsequence,

$$
\lim _{\rho \rightarrow 0} \rho \int_{0}^{\infty} e^{-\rho s}\left(\ell_{0}\left(z^{*}(s), \alpha^{*}(s)\right)\right) d s \geq \lim _{\rho \rightarrow 0} \rho w_{\rho}^{R}\left(z_{0}\right)=E^{R} \geq-E,
$$

and this implies thanks to (3.14) that

$$
\frac{1}{T_{0}+\tau} \int_{0}^{T_{0}+\tau} \ell_{0}\left(z^{*}(s), \alpha^{*}(s)\right) d s \geq-E
$$

Therefore,

$$
\int_{0}^{T_{0}} \ell_{0}(z(s), \alpha(s)) d s \geq-E\left(T_{0}+\tau\right)-\int_{T_{0}}^{T_{0}+\tau} \ell_{0}\left(z^{*}(s), \alpha^{*}(s)\right) d s \geq-E T_{0}-C .
$$




\subsection{Generalization of the previous results}

Let $R_{0}$ be a fixed real number such that $R_{0}>r_{0}$. It will prove useful to define the following open sets, see Figure 2:

$$
\begin{array}{r}
K_{i}=Z_{i} \cap\left\{x: x_{i}>R_{0}\right\}, \quad i=1, \ldots, N, \\
\widetilde{K}_{i}=Z_{i} \cap\left\{x: r_{0}<x_{i}<R_{0}\right\}, \quad i=1, \ldots, N, \\
\omega=\Omega \cap\left\{x: x_{i}<R_{0}, \forall i=1, \ldots, N\right\},
\end{array}
$$

with $Z_{i}$ defined in (1.2). Note that $\bar{\omega}=\cup_{i=0}^{N} \widetilde{\widetilde{K}}_{i}$ and that $\bar{\Omega}=\bar{\omega} \cup \bigcup_{i=1}^{N} \bar{K}_{i}$.

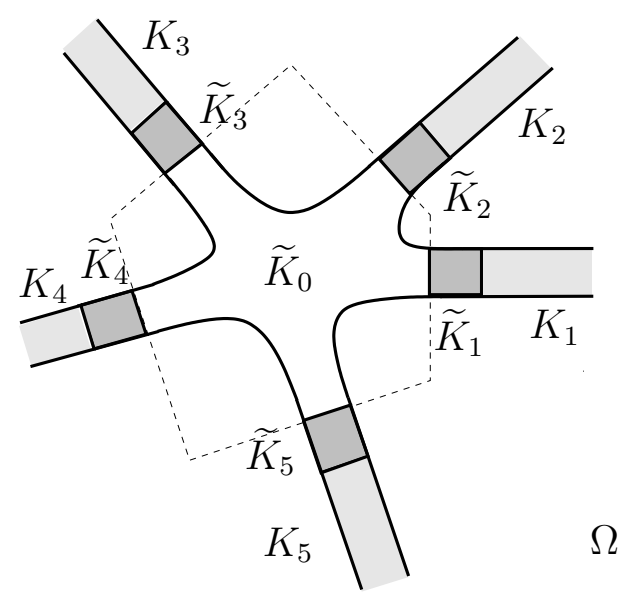

Figure 2: The set $\Omega$ is partitioned into different zones

Consider a subdomain $\tilde{\Omega}$ of $\Omega$ obtained as follows: call $\mathcal{I}$ a subset of $\{1, \ldots, N\}$ and define $\tilde{\Omega}$ by

$$
\overline{\tilde{\Omega}}=\overline{\widetilde{K}_{0}} \cup \bigcup_{i \in \mathcal{I}} \overline{\widetilde{K}_{i}} \cup \bigcup_{i \in \mathcal{I}} \overline{K_{i}}
$$

see Figure 3.

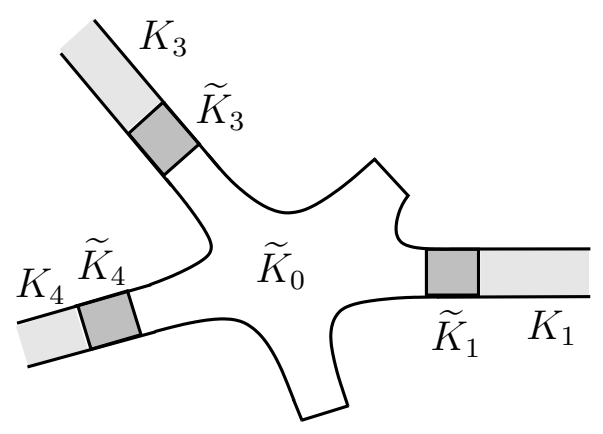

$\Omega_{p}$

Figure 3: The set $\tilde{\Omega}$ in the case when $\mathcal{I}=\{1,3,4\}$

Let $\tilde{\Omega}^{R}=\tilde{\Omega} \cap W^{R}$. As in $\S 3.1$, it is possible to obtain the existence of a unique ergodic 
constant $E_{\tilde{\Omega}}^{R}$ and of a bounded Lipschitz corrector $w_{\tilde{\Omega}}^{R}$ such that

$$
\begin{array}{ll}
H_{0}\left(\cdot, D w_{\tilde{\Omega}}^{R}\right) \geq E_{\tilde{\Omega}}^{R} & \text { in } \overline{\tilde{\Omega}^{R}}, \\
H_{0}\left(\cdot, D w_{\tilde{\Omega}}^{R}\right) \leq E_{\tilde{\Omega}}^{R} & \text { in } \tilde{\Omega}^{R},
\end{array}
$$

and $E_{\tilde{\Omega}}^{R} \leq E^{R} \leq E$ since $\tilde{\Omega}^{R} \subset \Omega^{R}$.

Remark 3.3. Remarks 3.1 and 3.2 hold with the obvious changes consisting of replacing $\Omega$, $\left(\right.$ resp. $\left.\Omega^{r}, \Omega^{R}, w^{R}, \tilde{w}^{R}\right)$ with $\tilde{\Omega},\left(\operatorname{resp} . \tilde{\Omega}^{r}, \tilde{\Omega}^{R}, w_{\tilde{\Omega}}^{R}, \tilde{w}_{\tilde{\Omega}}^{R}\right)$.

For $z_{0} \in \bar{\Omega}$, let $\mathcal{A}_{z_{0}}^{\tilde{\Omega}}$ be the set

$$
\mathcal{A}_{z_{0}}^{\tilde{\Omega}}=\left\{\alpha: \mathbb{R}_{+} \rightarrow A \text {, measurable, such that } \forall s \geq 0, z(s) \equiv z_{0}+\int_{0}^{s} \alpha(\theta) d \theta \in \bar{\Omega}\right\} .
$$

The following lemma is proved exactly as Lemma 3.1:

Lemma 3.2. There exists a constant $C$ such that for all $z_{0} \in \widetilde{K}_{0}$, (see (1.3) for the definition of $\left.\widetilde{K}_{0}\right)$, for all $\alpha \in \mathcal{A}_{z_{0}}^{\tilde{\Omega}}$ and $T_{0}>0$ such that $z\left(T_{0}\right) \in \widetilde{K}_{0}$, (recall that $\left.z(s) \equiv z_{0}+\int_{0}^{s} \alpha(\theta) d \theta\right)$,

$$
\int_{0}^{T_{0}} \ell_{0}(z(s), \alpha(s)) d s \geq-E T_{0}-C .
$$

\section{The main result: the effective problem and the convergence theorem}

The aim is first to introduce an effective Hamiltonian at the junction, noted $\bar{H}(O, \cdot)$ below and to define the effective problem. Then we will briefly summarize the results of Imbert-Monneau [20] on Hamilton-Jacobi equations on $\mathcal{G}$. Finally we will state the main convergence result.

\subsection{The effective Hamiltonian at the junction}

Let $\bar{H}_{i}^{+}\left(x_{i}, p_{i}\right)$ be defined by

$$
\bar{H}_{i}^{+}\left(x_{i}, p_{i}\right)=\sup _{\mu \in \mathcal{Z}_{i}^{+}}\left(-p_{i} \int_{[-1,1] \times A} a_{i} d \mu(y, a)-\ell_{i}^{r}(x, \mu)\right)
$$

where $\mathcal{Z}_{i}^{+}$is defined by $(2.16)$.

Remark 4.1. In the affine-convex case, $H_{i}^{+}$is characterized by

$$
\bar{H}_{i}^{+}\left(x_{i}, p_{i}\right)=\max _{a \in A, a_{i} \geq 0, a_{i}^{\perp}=0, y_{i}^{\perp} \in[-1,1]}\left(-p_{i} a_{i}-\ell_{i}\left(x_{i}, y_{i}^{\perp}, a\right)\right) .
$$

Let the function $\bar{H}(O, \cdot): \mathbb{R}^{N} \rightarrow \mathbb{R}$ be defined by

$$
\bar{H}(O, q)=\max _{i=1, \ldots, N} \bar{H}_{i}^{+}\left(0, q_{i}\right), \quad \forall q \in \mathbb{R}^{N},
$$

and the set of indices $\mathcal{I}(q)$ be defined by

$$
\mathcal{I}(q)=\left\{i: 1 \leq i \leq N, \bar{H}_{i}^{+}\left(0, q_{i}\right)=\bar{H}(O, q)\right\} .
$$


We also define what may be called the tangential Hamiltonian at the junction (see [2]) by

$$
\Lambda=\max _{i=1, \ldots, N} \Lambda_{i}^{0}
$$

where $\Lambda_{i}^{0}=\min _{p \in \mathbb{R}} \bar{H}_{i}(0, p)$.

Remark 4.2. From Lemma 2.1, we see that

$$
\bar{H}_{i}^{+}\left(0, p_{i}\right)= \begin{cases}\bar{H}_{i}\left(0, p_{i}\right)>\Lambda_{i}^{0} & \text { if } p_{i}<\underline{p}_{i}, \\ \Lambda_{i}^{0} & \text { if } p_{i} \geq \underline{p}_{i},\end{cases}
$$

then, it is clear that $\forall q \in \mathbb{R}^{N}$,

$$
\bar{H}(O, q) \geq \Lambda .
$$

In Figure 4, we give an example for the graphs of $p \mapsto \bar{H}_{i}(0, p)$ and of $p \mapsto \bar{H}_{i}^{+}(0, p)$. The constant $\Lambda_{i}^{0}$ is the minimal value of both $\bar{H}_{i}(0, \cdot)$ and $\bar{H}_{i}^{+}(0, \cdot)$.

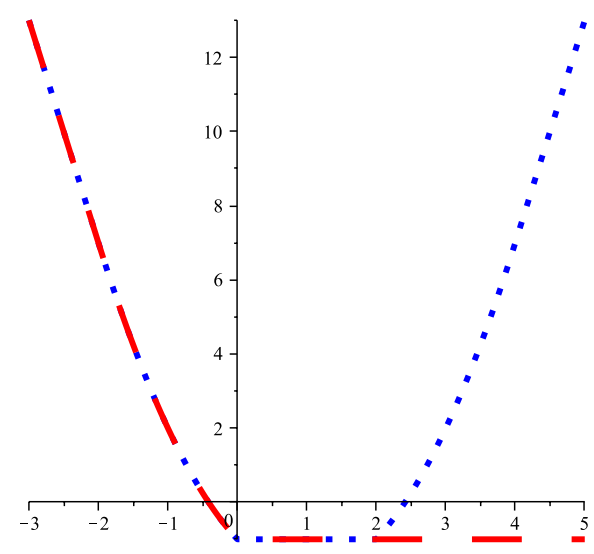

Figure 4: The graphs of the Hamiltonian $p \mapsto \bar{H}_{i}(0, p)$ and of $p \mapsto \bar{H}_{i}^{+}(0, p)$ coincide for $p \leq \bar{p}_{i}$, and $\bar{H}_{i}^{+}(0, p)=\Lambda_{i}^{0}$ for $p \geq \underline{p}_{i}$. In the example, $\underline{p}_{i}=0$ and $\bar{p}_{i}=2$.

Lemma 4.1. Under Assumptions 1.1- 1.3 and 2.1, $\Lambda$ defined in (4.5) and $E$ defined in (3.10) satisfy

$$
\Lambda \leq E .
$$

Proof. For any $i=1, \ldots, N$, choose $p_{i} \in \operatorname{argmin} \bar{H}_{i}(0, \cdot)$, and $L_{i}$ as in Assumption 2.1. Take $z_{0, i}>2 r_{0}+L_{i}, z_{0}=z_{0, i} e_{i}+z_{0, i}^{\perp} e_{i}^{\perp} \in \bar{\Omega}$ and any $R>z_{i}+L_{i}$. From Assumption 2.1 and Remark 2.2, we know that for all $t>0$, there exists a control law $\alpha \in \mathcal{A}_{z_{0}}$ such that for all $s \in[0, t], z(s)=z_{0}+\int_{0}^{s} \alpha(\theta) d \theta$ belongs to the set $\left\{x: x_{i} \in\left[r_{0}, z_{0, i}+L_{i}\right], x_{i}^{\perp} \in[-1,1]\right\} \subset \bar{\Omega}^{R}$ and

$$
\int_{0}^{t} \ell_{0}(z(s), \alpha(s)) d s \leq-t \bar{H}_{i}\left(0, p_{i}\right)+C,
$$

for a constant $C$ independent of $t$.

From Assumption 1.1, there exists a finite time $\tau>0$ (which is bounded uniformly w.r.t. $t$ ) and $a \in A$ such that $z(t)+a \tau=z_{0}$. The following control law

$$
\alpha^{*}(s)= \begin{cases}\alpha(s) & \text { if } s \in[0, t], \\ a & \text { if } s \in(t, t+\tau],\end{cases}
$$


can be extended by periodicity to $\mathbb{R}^{+}$(the period is $\left.t+\tau\right)$ to yield a periodic trajectory $z^{*}(s) \equiv$ $z_{0}+\int_{0}^{s} \alpha^{*}(\theta) d \theta$, which stays in $\bar{\Omega}^{R}$. The arguments contained in the proof of Lemma 3.1 can then be repeated to obtain that

$$
\frac{1}{t+\tau} \int_{0}^{t+\tau} \ell_{0}\left(z^{*}(s), \alpha^{*}(s)\right) d s \geq-E
$$

and that

$$
\int_{0}^{t} \ell_{0}(z(s), \alpha(s)) d s \geq-E t-\tilde{C}
$$

for another constant $\tilde{C}$. Combining this with (4.9) yields that $-E t-\tilde{C} \leq-t \bar{H}_{i}\left(0, p_{i}\right)+C=$ $-t \Lambda_{i}^{0}+C$. Since $t$ can be chosen as large as desired, we get that $\Lambda_{i}^{0} \leq E$, and since the argument above can be repeated for all $i=1, \ldots, N$, we obtain (4.8).

\subsection{The effective problem on $\mathcal{G}$}

Here we introduce the effective problem on the network $\mathcal{G}$ and define its viscosity solution. We also recall some of the results obtained by Imbert and Monneau [20], in a presentation adapted to the context.

The effective Hamilton-Jacobi equation on $\mathcal{G}$ is:

$$
\begin{aligned}
\lambda u(x)+\bar{H}_{i}\left(x_{i}, D u(x)\right) & =0 \quad x=x_{i} e_{i} \in G_{i}, \\
\lambda u(O)+\max (E, \bar{H}(O, D u(O))) & =0 \quad x=O,
\end{aligned}
$$

where $\bar{H}(O, \cdot)$ is defined in (4.3), $E$ is defined in (3.10) and

$$
D u(x)= \begin{cases}\frac{d u}{d x_{j}}(x) & \text { if } x \in G_{i}, \\ \left(\frac{d u}{d x_{1}}(O), \ldots, \frac{d u}{d x_{N}}(O)\right) & \text { if } x=O .\end{cases}
$$

\subsubsection{Test functions}

For the definition of viscosity solutions on the irregular set $\mathcal{G}$, it is necessary to first define a class of the admissible test functions

Definition 4.1. A function $\varphi: \mathcal{G} \rightarrow \mathbb{R}$ is an admissible test function if

- $\varphi$ is continuous in $\mathcal{G}$ and $\mathcal{C}^{1}$ in $\mathcal{G} \backslash\{O\}$

- for any $j, j=1, \ldots, N,\left.\varphi\right|_{\bar{G}_{j}} \in \mathcal{C}^{1}\left(\bar{G}_{j}\right)$.

The set of admissible test function is noted $\mathcal{R}(\mathcal{G})$. If $\varphi \in \mathcal{R}(\mathcal{G})$ and $\zeta \in \mathbb{R}$, let $D \varphi\left(x, \zeta e_{i}\right)$ be defined by $D \varphi\left(x, \zeta e_{i}\right)=\zeta \frac{d \varphi}{d x_{i}}(x)$ if $x \in G_{i} \backslash\{O\}$ and $D \varphi\left(O, \zeta e_{i}\right)=\zeta \lim _{h \rightarrow 0+} \frac{d \varphi}{d x_{i}}\left(h e_{i}\right)$.

Property 4.1. If $\varphi=g \circ \psi$ with $g \in \mathcal{C}^{1}$ and $\psi \in \mathcal{R}(\mathcal{G})$, then $\varphi \in \mathcal{R}(\mathcal{G})$ and

$$
D \varphi(O, \zeta)=g^{\prime}(\psi(O)) D \psi(O, \zeta) \text {. }
$$




\subsubsection{Definition of viscosity solutions}

Definition 4.2. $\quad$ - An upper semi-continuous function $u: \mathcal{G} \rightarrow \mathbb{R}$ is a viscosity subsolution of (4.10-4.11) in $\mathcal{G}$ if for any $x \in \mathcal{G}$, any $\varphi \in \mathcal{R}(\mathcal{G})$ s.t. $u-\varphi$ has a local maximum point at $x$, then

$$
\begin{aligned}
& \lambda u(x)+\bar{H}_{i}\left(x, \frac{d \varphi}{d x_{i}}(x)\right) \leq 0 \\
& \lambda u(O)+\max \left(E, \bar{H}\left(O, \frac{d \varphi}{d x_{1}}(O), \ldots, \frac{d \varphi}{d x_{N}}(O)\right)\right) \leq 0 .
\end{aligned}
$$

- A lower semi-continuous function $u: \mathcal{G} \rightarrow \mathbb{R}$ is a viscosity supersolution of (4.10-4.11) if for any $x \in \mathcal{G}$, any $\varphi \in \mathcal{R}(\mathcal{G})$ s.t. $u-\varphi$ has a local minimum point at $x$, then

$$
\begin{array}{ll}
\lambda u(x)+\bar{H}_{i}\left(x, \frac{d \varphi}{d x_{i}}(x)\right) \geq 0 & \text { if } x \in G_{i}, \\
\lambda u(O)+\max \left(E, \bar{H}\left(O, \frac{d \varphi}{d x_{1}}(O), \ldots, \frac{d \varphi}{d x_{N}}(O)\right)\right) \geq 0 . &
\end{array}
$$

- We say that $u$ is a viscosity solution of (4.10-4.11) in $\mathcal{G}$ if it is both a viscosity subsolution and supersolution of (4.10-4.11).

\subsubsection{Comparison principle}

The following result is a consequence of the general comparison results proved in [20]:

Theorem 4.1. Under the Assumptions 1.1- 1.3, for all viscosity subsolution $u$ and all supersolution $v$ of (4.10-4.11) satisfying

$$
u(x) \leq C(1+|x|) \quad \text { and } \quad v(x) \geq-C(1+|x|)
$$

for some positive number $C$, we have

$$
u \leq v \quad \text { in } \mathcal{G} \text {. }
$$

\subsection{The convergence result}

We are ready to the state the main result of the paper:

Theorem 4.2 (Convergence). Under Assumptions 1.1-1.3 and 2.1, as $\varepsilon \rightarrow 0+$, the functions $u_{\varepsilon}$ converge locally uniformly to the unique viscosity solution $u$ of (4.10)-(4.11).

The proof of this Theorem is given in Section 6. It is based on the perturbed test-function method of Evans. The perturbed test-functions involve suitable correctors which are constructed in Section 5.

\section{Correctors at the junction when $\bar{H}(O, p)>E$}

The aim here is to construct correctors (in unbounded domains) which will be used in $\S 6$ in the perturbed test-function method of Evans, [17]. The strategy for constructing the correctors is reminiscent of the one used by Ishii in [22], although a new difficulty arises from the unboundedness of the domains in which the correctors will be defined. 
Remark 5.1. The question of the correctors in unbounded domains has very recently been addressed by P-L. Lions in his lectures at Collège de France, [23], precisely in january and february 2014 (therefore after the completion of the present work): the lectures dealt with recent and still unpublished results obtained in collaboration with T. Souganidis on the asymptotic behavior of solutions of Hamilton-Jacobi equations in a periodic framework with some localized defects. In this context, P-L. Lions addressed similar phenomena as those mentioned below, namely the possible nonexistence of correctors.

Let $p \in \mathbb{R}^{N}$ be such that $\bar{H}(O, p)>\Lambda$. Then from (4.6), we see that for any index $i \in \mathcal{I}(p)$, $p_{i}<\underline{p}_{i}$ and $\bar{H}(O, p)=\bar{H}_{i}^{+}\left(0, p_{i}\right)=\bar{H}_{i}\left(0, p_{i}\right)$.

Take $R_{0}>r_{0}$ as in $\S 3.4$ and let $\psi_{p}$ be a smooth function on $\bar{\Omega}$ such that

$$
\begin{array}{lll}
\psi_{p}(z)=p_{i} z_{i} & & \forall z \in \bar{K}_{i}, \\
\psi_{p}(z)=p_{i} \Psi\left(z_{i}\right) & & \forall z \in \widetilde{K}_{i}, \\
\psi_{p}(z)=0 & & \forall z \in \widetilde{K}_{0},
\end{array}
$$

where $\Psi$ is a smooth non decreasing function defined on $\left[r_{0}, R_{0}\right]$ such that $\Psi=0$ in a neighborhood of $r_{0}, \Psi(r)=r$ in a neighborhood of $R_{0}$, and $\Psi(r) \leq r$ for all $r \in\left[r_{0}, R_{0}\right]$.

Let the open connected set $\Omega_{p}$ be defined by

$$
\bar{\Omega}_{p}=\overline{\widetilde{K}_{0}} \cup \bigcup_{i \in \mathcal{I}(p)} \overline{\widetilde{K}_{i}} \cup \bigcup_{i \in \mathcal{I}(p)} \overline{K_{i}}
$$

where $\widetilde{K}_{0}, \widetilde{K}_{i}$ and $K_{i}$ are defined respectively in (1.3), (3.16) and (3.15), see Figure 5 . Note that $\bar{\Omega}_{p}$ corresponds to a set $\tilde{\Omega}$ defined in $\S 3.4$.

Below, a corrector associated to $p$ will be defined in $\bar{\Omega}_{p}$. Its existence will be stated in Theorem 5.1. Lemmas 5.1, 5.2 and 5.3 below are the main steps for proving Theorem 5.1.

Remark 5.2. The idea of truncating the domain and use $\Omega_{p}$ instead of $\Omega$ comes from the fact that we need a bounded corrector, and constructing a bounded corrector does not seem possible in the full domain $\bar{\Omega}$. Since the correctors are defined in subdomains, the method of the perturbed test functions of Evans will have to be suitably modified, see $\S 6$.

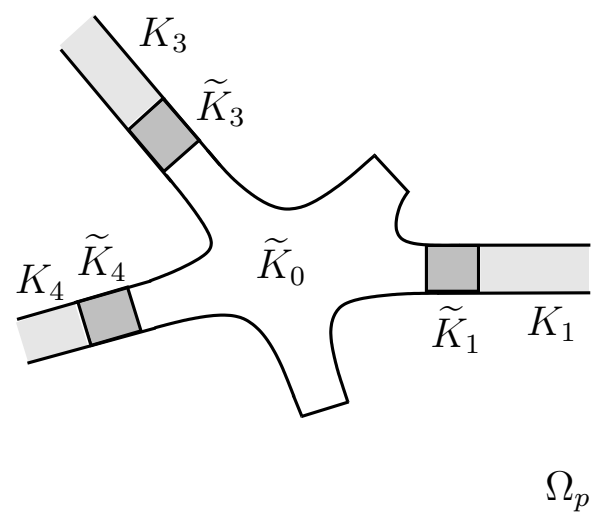

Figure 5: The set $\Omega_{p}$ in the case when $\mathcal{I}(p)=\{1,3,4\}$

For $z_{0} \in \bar{\Omega}_{p}$ and $t \geq 0$, let $u\left(z_{0}, t\right)$ be defined by

$$
u\left(z_{0}, t\right)=\inf _{\alpha \in \mathcal{A}_{y}^{p}} J\left(z_{0}, t, \alpha\right),
$$


where

$$
J\left(z_{0}, t, \alpha\right)=\int_{0}^{t}\left(D \psi_{p}(z(s)) \cdot \alpha(s)+\ell_{0}(z(s), \alpha(s))\right) d s
$$

and

$$
\begin{aligned}
& \dot{z}(s)=\alpha(s), \quad s>0, \quad z(0)=z_{0}, \\
& \mathcal{A}_{z_{0}}^{p}=\left\{\alpha: \alpha(s) \in A \text { for a.a. } s ; z(s) \in \bar{\Omega}_{p} \text { for all } s\right\} .
\end{aligned}
$$

It is well known, see [9], that $u$ is a Lipschitz continuous viscosity solution of

$$
\begin{array}{ll}
\frac{\partial u}{\partial t}(z, t)+H_{0}\left(z, D \psi_{p}+D u\right) \geq 0 & \text { in } \bar{\Omega}_{p} \times \mathbb{R}_{+}, \\
\frac{\partial u}{\partial t}(z, t)+H_{0}\left(z, D \psi_{p}+D u\right) \leq 0 & \text { in } \Omega_{p} \times \mathbb{R}_{+},
\end{array}
$$

where

$$
H_{0}(z, q)=\max _{a \in A}\left(-q \cdot a-\ell_{0}(z, a)\right) .
$$

Lemma 5.1. Let $p \in \mathbb{R}^{N}$ be such that $\bar{H}(O, p)>\Lambda$. Under Assumptions 1.1-1.3 and 2.1, there exists a constant $C$ such that for all $z \in \bar{\Omega}_{p}$ and $t \geq 0$,

$$
u(z, t) \leq-t \bar{H}(O, p)+C .
$$

Proof. Consider $z_{0} \in \bar{\Omega}_{p}$. We make out several cases:

Case 1 There exists $i$ in $\mathcal{I}(p)$ such that $z_{0} \in K_{i}$ and $z_{0, i}>L_{i}+R_{0}$ where $L_{i}$ is the constant (possibly depending on $p_{i}$ ) appearing in Assumption 2.1. Consider the control law $\tilde{\alpha}$ described in Assumption 2.1 with $y_{0}=z_{0, i}^{\perp}$. This control law is such that the corresponding trajectory $\tilde{z}(s)=z_{0}+\int_{0}^{s} \tilde{\alpha}(\theta) d \theta$ remains in $\bar{K}_{i}$ for all $s \in[0, t]$. Moreover,

$$
J\left(z_{0}, t, \tilde{\alpha}\right)=\int_{0}^{t} p_{i} \tilde{\alpha}_{i}(s)+\ell_{i}\left(0, \tilde{z}_{i}^{\perp}(s), \tilde{\alpha}(s)\right) d s \leq-t \bar{H}(O, p)+C_{i, 2},
$$

from (2.22). Hence

$$
u\left(z_{0}, t\right) \leq-t \bar{H}(O, p)+C_{i, 2} .
$$

Case 2 In the opposite case, there exists a constant $\tilde{L}$ (depending on $p$ ) such that $\left|z_{0}\right| \leq \tilde{L}$. Let $i_{0}$ be an index $i_{0} \in \mathcal{I}(p)$ minimizing distance $\left(z_{0}-L_{j} e_{j}, \bar{K}_{j}\right)$. From the controllability assumption 1.1, it is possible to find $T>0$ such that for all $z_{0}$ in Case 2, there exists a control law $\bar{\alpha}$ such that $\bar{z}(\tau)=z_{0}+\int_{0}^{\tau} \bar{\alpha}(s) d s \in \bar{K}_{i_{0}}, \bar{z}_{i_{0}}(\tau)>L_{i_{0}}+R_{0}$ for some $\tau \leq T$ and $\bar{z}(s) \in \bar{\Omega}_{p}$ for all $s \in[0, \tau]$.

It is possible to extend $\bar{\alpha}$ by $s \mapsto \tilde{\alpha}(s-\tau)$ for $t-\tau>s>\tau$ where $\tilde{\alpha}$ is the control described in Assumption 2.1 for $i=i_{0}, t=t-\tau$ and $y_{0}=\bar{z}_{0, i}^{\perp}(\tau)$.

It is easy to see that the related trajectory remains in $\bar{\Omega}_{p}$ and that there exists a constant $C$ independent of $z_{0}$ in Case 2 such that

$$
J\left(z_{0}, t, \bar{\alpha}\right)=\int_{0}^{t} D \psi_{p}(\bar{z}(s)) \cdot \bar{\alpha}(s)+\ell_{0}(\bar{z}(s), \bar{\alpha}(s)) d s \leq-t \bar{H}(O, p)+C,
$$

which achieves the proof. 
Lemma 5.2. Let $p \in \mathbb{R}^{N}$ be such that $\bar{H}(O, p)>\Lambda$. There exists a constant $c \geq 0$ such that for all $z_{0} \in \bar{\Omega}_{p}$ and $t \geq 0$,

$$
u\left(z_{0}, t\right) \geq-t \max (E, \bar{H}(O, p))-c .
$$

Proof. For any $\varepsilon>0$, we consider $\alpha \in \mathcal{A}_{z_{0}}^{p}$ such that $J\left(z_{0}, t, \alpha\right) \leq u\left(z_{0}, t\right)+\varepsilon$. Call $z(s)=$ $z_{0}+\int_{0}^{s} \alpha(\theta) d \theta$. In what follows, $c$ is a positive constant possibly depending on $p$ that may change from line to line.

- Assume first that $z_{0} \in \widetilde{K}_{0}$. We make out two cases:

- If $z(t) \in \overline{\widetilde{K}}_{0}$, it is possible to modify the control law as in the Lemma 3.2 and Lemma 3.1 to obtain a $t+\tau$-periodic trajectory $z^{*}(\cdot)$. Then $s \mapsto \psi_{p}\left(z^{*}((s))\right.$ is a function belonging to $W_{0}^{1, \infty}(0, t+\tau)$, whose weak derivative coincides almost everywhere with $s \mapsto D \psi_{p}\left(z^{*}(s)\right) \cdot \alpha^{*}(s)$. Therefore $\int_{0}^{t+\tau} D \psi_{p}\left(z^{*}(s)\right) \cdot \alpha^{*}(s) d s=\psi_{p}\left(z^{*}(t+\tau)\right)-$ $\psi_{p}\left(z^{*}(0)\right)=0$, then

$$
J\left(z_{0}, t+\tau, \alpha^{*}\right)=\int_{0}^{t+\tau} \ell_{0}\left(z^{*}(s), \alpha^{*}(s)\right) d s \geq-E t-c .
$$

Thus,

$$
J\left(z_{0}, t+\tau, \alpha^{*}\right)=J\left(z_{0}, t, \alpha\right)+J\left(z(t), \tau, \alpha^{*}(\cdot+t)\right.
$$

and $J\left(z(t), \tau, \alpha^{*}(\cdot+t)\right.$ is bounded by a constant thanks to the definition of $\psi_{p}$ for trajectories lying in $\widetilde{K}_{0}$.

Therefore,

$$
J\left(z_{0}, t, \alpha\right)=J\left(z_{0}, t+\tau, \alpha^{*}\right)-J\left(z(t), \tau, \alpha^{*}(\cdot+t) \geq-E t-c .\right.
$$

- $z(t) \in\left(\bar{K}_{i} \cup \overline{\widetilde{K}}_{i}\right) \backslash \overline{\widetilde{K}}_{0}$ for some $i \in \mathcal{I}(p)$. Let $\bar{t}$ be the supremum of all entry times in $\bar{K}_{i} \cup \widetilde{K}_{i}$ smaller than $t$. Then $z(\bar{t}) \in \widetilde{K}_{0}$ and we can repeat the argument above to prove that

$$
J\left(z_{0}, \bar{t}, \alpha\right) \geq-E \bar{t}-c .
$$

There only remains to study $\int_{\bar{t}}^{t}\left(D \psi_{p}(z(s)) \cdot \alpha(s)+\ell_{0}(z(s), \alpha(s))\right) d s$. For $s \in[\bar{t}, t]$, $\ell_{0}(z(s), \alpha(s))=\ell_{i}\left(0, z_{i}^{\perp}(s), \alpha(s)\right)$. Moreover, $\left|\int_{\bar{t}}^{t} D \psi_{p}(z(s)) \cdot \alpha(s) d s-p_{i}\left(z_{i}(t)-z_{i}(\bar{t})\right)\right| \leq$ c. Hence,

$$
\left|\int_{\bar{t}}^{t}\left(D \psi_{p}(z(s)) \cdot \alpha(s)+\ell_{0}(z(s), \alpha(s))\right) d s-\int_{\bar{t}}^{t}\left(p_{i} \alpha_{i}(s)+\ell_{i}\left(0, z_{i}^{\perp}(s), \alpha(s)\right)\right) d s\right| \leq c .
$$

But

$$
\int_{\bar{t}}^{t}\left(p_{i} \alpha_{i}(s)+\ell_{i}\left(0, z_{i}^{\perp}(s), \alpha(s)\right)\right) d s \geq v_{i}\left(0, p_{i}, z_{i}^{\perp}(\bar{t}), t-\bar{t}\right)
$$

where $v_{i}$ is defined in (2.8). From (2.9),

$$
\int_{\bar{t}}^{t}\left(p_{i} \alpha_{i}(s)+\ell_{i}\left(0, z_{i}^{\perp}(s), \alpha(s)\right)\right) d s \geq-(t-\bar{t}) \bar{H}_{i}\left(0, p_{i}\right)-c .
$$

Summing all the contributions, we get that

$$
J\left(z_{0}, t, \alpha\right) \geq(t-\bar{t})\left(-\bar{H}_{i}\left(0, p_{i}\right)\right)-E \bar{t}-c .
$$

But $\bar{H}_{i}\left(0, p_{i}\right)=\bar{H}(O, p)$ since $i \in \mathcal{I}(p)$. Hence

$$
J\left(z_{0}, t, \alpha\right) \geq(t-\bar{t})(-\bar{H}(O, p))-E \bar{t}-c \geq \min (-\bar{H}(O, p),-E) t-c .
$$


- Assume now that $z_{0} \in \bar{K}_{i} \cup \overline{\widetilde{K}}_{i}$ for some $i \in \mathcal{I}(p)$. We make out two cases:

- If the trajectory stays in $\bar{K}_{i} \cup \widetilde{\widetilde{K}}_{i}$, then

$$
\begin{aligned}
J\left(z_{0}, t, \alpha\right) & =\psi_{p}(z(t))-\psi_{p}\left(z_{0}\right)+\int_{0}^{t} \ell_{i}\left(0, z_{i}^{\perp}(s), \alpha(s)\right) d s \\
& \geq p_{i}\left(z_{i}(t)-z_{0, i}\right)-c+\int_{0}^{t} \ell_{i}\left(0, z_{i}^{\perp}(s), \alpha(s)\right) d s .
\end{aligned}
$$

Therefore, $J\left(z_{0}, t, \alpha\right) \geq v_{i}\left(0, z_{0, i}^{\perp}, t\right)-c \geq-t \bar{H}(0, p)-c$.

- The trajectory $z$ leaves $\bar{K}_{i} \cup \overline{\widetilde{K}}_{i}$. Let $\theta$ be the smallest time such that $z(\theta) \in \overline{\widetilde{K}}_{0}$. Then

$$
J\left(z_{0}, t, \alpha\right)=J\left(z_{0}, \theta, \alpha\right)+J(z(\theta), t-\theta, \tilde{\alpha}),
$$

where $\tilde{\alpha}(s)=\alpha(s-\theta)$. We have just seen that $J\left(z_{0}, \theta, \alpha\right) \geq-\theta \bar{H}(O, p)-c$. From the previous case (when $\left.z_{0} \in \widetilde{K}_{0}\right), J(z(\theta), t-\theta, \tilde{\alpha}) \geq(t-\theta) \min (-\bar{H}(O, p),-E)-c$. Thus, $J\left(z_{0}, t, \alpha\right) \geq t \min (-\bar{H}(O, p),-E)-c$.

The result follows letting $\varepsilon \rightarrow 0$.

As a consequence of Lemmas 5.1 and 5.2, and using also that $E \geq \Lambda$ from Lemma 4.1, we obtain the following:

Lemma 5.3. Let $p \in \mathbb{R}^{N}$ be such that $\bar{H}(O, p)>E$. Under Assumptions 1.1-1.3 and 2.1, the function v: $(z, t) \mapsto u(z, t)+t \bar{H}(O, p)$ is bounded and Lipschitz continuous on $\bar{\Omega}_{p} \times \mathbb{R}_{+}$. It is a viscosity solution of

$$
\begin{array}{lc}
\frac{\partial v}{\partial t}(z, t)+H_{0}\left(z, D \psi_{p}+D v\right)-\bar{H}(O, p) \geq 0 & \text { in } \bar{\Omega}_{p} \times \mathbb{R}_{+}, \\
\frac{\partial v}{\partial t}(z, t)+H_{0}\left(z, D \psi_{p}+D v\right)-\bar{H}(O, p) \leq 0 & \text { in } \Omega_{p} \times \mathbb{R}_{+} .
\end{array}
$$

Define the function $w$ by

$$
w(z, t)=\inf _{r>0} v(z, t+r) .
$$

It is easy to check that the function $w$ is bounded and Lipschitz continuous. It can also be seen that $w$ is a non decreasing function of time. From e.g. [9], Proposition 2.11, page 302, or [22], Proposition 1.10, page 125, $w$ is a viscosity supersolution of

$$
\frac{\partial w}{\partial t}(z, t)+H_{0}\left(z, D \psi_{p}+D w\right)-\bar{H}(O, p) \geq 0 \quad \text { in } \bar{\Omega}_{p} \times \mathbb{R}_{+} .
$$

From the convexity of $H_{0}(z, \cdot)$, we get the following Lemma

Lemma 5.4. $w$ is a viscosity subsolution of

$$
\frac{\partial w}{\partial t}(z, t)+H_{0}\left(z, D \psi_{p}+D w\right)-\bar{H}(O, p) \leq 0 \quad \text { in } \Omega_{p} \times \mathbb{R}_{+} .
$$

Proof. See [22] Lemma 5.11. Two possible arguments may be used, the first one from Barron and Jensen[13], and the second one from Barles[10]. 
Let $\chi_{p}(z)$ be defined by $\chi_{p}(z)=\lim _{t \rightarrow \infty} w(z, t)=\sup _{t} w(z, t)$. It is obvious that $\chi_{p}$ is bounded. The convergence of $w(z, t)$ to $\chi_{p}(z)$ is uniform on the bounded subsets of $\bar{\Omega}_{p}$. Therefore $\chi_{p}$ is a Lipschitz function on $\bar{\Omega}_{p}$. Hence, $\chi_{p}$ as a function of $z$ and $t$ is a viscosity solution of (5.12) and (5.13). Since it does not depend on $t, \chi_{p}$ is a bounded and Lipschitz solution of

$$
\begin{array}{ll}
H_{0}\left(z, D \psi_{p}+D \chi_{p}\right)-\bar{H}(O, p) \geq 0 & \text { in } \bar{\Omega}_{p}, \\
H_{0}\left(z, D \psi_{p}+D \chi_{p}\right)-\bar{H}(O, p) \leq 0 & \text { in } \Omega_{p} .
\end{array}
$$

Therefore, the following theorem has been obtained:

Theorem 5.1. Let $p \in \mathbb{R}^{N}$ be such that $\bar{H}(O, p)>E$. Under Assumptions 1.1-1.3 and 2.1, there exists a bounded and Lipschitz function $\chi_{p}$ defined on $\bar{\Omega}_{p}$ which is a viscosity solution of (5.14)-(5.15).

\section{Convergence}

The aim is to study the asymptotic behavior of the sequence of functions $\left(u_{\varepsilon}\right)$ as $\varepsilon \rightarrow 0+$ where $u_{\varepsilon}$ is the unique viscosity solution of (1.5) (1.6).

A sequence of continuous functions $\left(v_{\varepsilon}\right)$ defined on $\Omega_{\varepsilon}$ is said to converge locally uniformly to a continuous function $v$ defined on $\mathcal{G}$ if for all $M>0$,

$$
\lim _{\varepsilon \rightarrow 0} \sup _{x \in \mathcal{G}, y \in \Omega_{\varepsilon} \cap B(O, M),|y-x| \leq \varepsilon}\left|v(x)-v_{\varepsilon}(y)\right|=0 .
$$

Theorem 6.1. Under Assumptions 1.1-1.3 and 2.1, consider the weak limits in the viscosity sense, or relaxed semilimits, of $u_{\varepsilon}$, as $\varepsilon \rightarrow 0+:$ for all $x \in \mathcal{G}$,

$$
\underline{u}(x)=\liminf _{\varepsilon \rightarrow 0+, x^{\prime} \rightarrow x, x^{\prime} \in \Omega_{\varepsilon}} u_{\varepsilon}\left(x^{\prime}\right) ; \quad \bar{u}(x)=\limsup _{\varepsilon \rightarrow 0+, x^{\prime} \rightarrow x, x^{\prime} \in \Omega_{\varepsilon}} u_{\varepsilon}\left(x^{\prime}\right) .
$$

Then, $\underline{u}$ is a bounded supersolution of

$$
\begin{array}{lll}
\lambda \underline{u}(x)+\bar{H}(x, D \underline{u}(x)) & \geq 0, & \text { if } x \in \mathcal{G} \backslash\{O\}, \\
\lambda \underline{u}(O)+\max (E, \bar{H}(O, D \underline{u}(O))) & \geq 0, & \text { if } x=O,
\end{array}
$$

and $\bar{u}$ is a bounded subsolution of

$$
\begin{array}{lll}
\lambda \bar{u}(x)+\bar{H}(x, D \bar{u}(x)) & \leq 0, & \text { if } x \in \mathcal{G} \backslash\{O\}, \\
\lambda \bar{u}(O)+\max (E, \bar{H}(O, D \bar{u}(O))) & \leq 0, & \text { if } x=O .
\end{array}
$$

Proof. Recall that $u_{\varepsilon}$ is bounded by a constant $M$ independent of $\varepsilon$ from the assumptions on $\ell_{\varepsilon}$. Therefore $\underline{u}$ and $\bar{u}$ are bounded. We first prove that $\bar{u}$ is a subsolution of (6.2).

$\bar{u}$ is a subsolution of (6.2) To prove that $\bar{u}$ is a subsolution of (6.2), we consider a strict maximum $\bar{x}$ of $\bar{u}-\varphi$, where $\varphi$ is a regular test-function for (6.1)-(6.2). We may assume that $\varphi(\bar{x})=\bar{u}(\bar{x})$.

We want to show that

$$
\begin{array}{lll}
\lambda \varphi(\bar{x})+\bar{H}(\bar{x}, D \varphi(\bar{x})) & \leq 0, & \text { if } \bar{x} \in \mathcal{G} \backslash\{O\}, \\
\lambda \varphi(O)+\max (E, \bar{H}(O, D \varphi(O))) & \leq 0, & \text { if } \bar{x}=O .
\end{array}
$$


We just have to focus on the case when $\bar{x}=O$, because if $\bar{x} \neq O,(6.3)$ is proved in [3]. Suppose by contradiction that

$$
\lambda \varphi(O)+\max (E, \bar{H}(O, D \varphi(O)))=\gamma>0 .
$$

Let $p \in \mathbb{R}^{N}$ be defined by $p=\left(D \varphi\left(O, e_{1}\right), \ldots, D \varphi\left(O, e_{N}\right)\right)$. We make out two cases:

First case: $\bar{H}(O, p)>E$. In this case (6.4) becomes:

$$
\lambda \varphi(O)+\bar{H}(O, D \varphi(O))=\gamma>0 .
$$

For $\Omega_{p}$ defined in (5.2), let the auxiliary function $\xi_{\varepsilon}$ be defined in $\varepsilon \bar{\Omega}_{p}$ by

$$
\begin{array}{lll}
\xi_{\varepsilon}(x)=\varphi\left(x_{i} e_{i}\right)-\varphi(O) & & \forall x \in \varepsilon \bar{K}_{i}, i \in \mathcal{I}(p), \\
\xi_{\varepsilon}(x)=\frac{\varepsilon}{x_{i}}\left(\varphi\left(x_{i} e_{i}\right)-\varphi(O)\right) \Psi\left(\frac{x}{\varepsilon}\right) & & \forall x \in \varepsilon \widetilde{K}_{i}, i \in \mathcal{I}(p), \\
\xi_{\varepsilon}(x)=0 & & \forall x \in \varepsilon \widetilde{K}_{0},
\end{array}
$$

where $\Psi$ is the same function as the one used in (5.1). Note that the function $x \mapsto$ $\xi_{\varepsilon}(x)-\varepsilon \psi_{p}\left(\frac{x}{\varepsilon}\right)$ is a $\mathcal{C}^{1}$ function which vanishes in $\varepsilon \widetilde{K}_{0}$ and that

$$
\lim _{\rho \rightarrow 0} \sup _{0<\varepsilon<\frac{\rho}{R_{0}}}\left\|D \xi_{\varepsilon}(x)-D_{z} \psi_{p}\left(\frac{x}{\varepsilon}\right)\right\|_{L^{\infty}\left(\varepsilon \bar{\Omega}_{p} \cap B_{\rho}(O)\right)}=0,
$$

where $R_{0}$ is the number fixed at the beginning of $\S 5$. Indeed,

$$
\begin{array}{rlrl}
D \xi_{\varepsilon}(x) & =\frac{d \varphi}{d x_{i}}\left(x_{i} e_{i}\right) e_{i}=p_{i} e_{i}+o(1)=D_{z} \psi_{p}\left(\frac{x}{\varepsilon}\right)+o(1) & & \forall x \in \varepsilon \bar{K}_{i}, \\
D \xi_{\varepsilon}(x) & =\frac{\varphi\left(x_{i} e_{i}\right)-\varphi(O)}{x_{i}} \frac{\partial \Psi}{\partial z_{i}}\left(\frac{x}{\varepsilon}\right) e_{i}-\frac{\varepsilon}{x_{i}}\left(\frac{\varphi\left(x_{i} e_{i}\right)-\varphi(O)}{x_{i}}-\frac{d \varphi}{d x_{i}}\left(x_{i} e_{i}\right)\right) \Psi\left(\frac{x}{\varepsilon}\right) e_{i} & \\
& =\left(p_{i}+o(1)\right) \frac{\partial \Psi}{\partial z_{i}}\left(\frac{x}{\varepsilon}\right) e_{i}+\frac{\varepsilon}{x_{i}} \Psi\left(\frac{x}{\varepsilon}\right) o(1) e_{i} & \\
& =D_{z} \psi_{p}\left(\frac{x}{\varepsilon}\right)+o(1) & & \forall x \in \varepsilon \widetilde{K}_{i}, \\
D \xi_{\varepsilon}(x) & =0 & & \forall x \in \varepsilon \widetilde{K}_{0},
\end{array}
$$

where $o(1)$ means a family of functions $\left(f_{\varepsilon}\right)_{\varepsilon}$ defined in $\varepsilon \bar{\Omega}_{p}$ such that

$$
\lim _{\rho \rightarrow 0} \sup _{0<\varepsilon<\frac{\rho}{R_{0}}}\left\|f_{\varepsilon}\right\|_{L^{\infty}\left(\varepsilon \bar{\Omega}_{p} \cap B_{\rho}(O)\right)}=0 .
$$

Define the perturbed test-function

$$
\varphi_{\varepsilon}(x)=\varphi(O)+\xi_{\varepsilon}(x)+\varepsilon \chi_{p}\left(\frac{x}{\varepsilon}\right)
$$

in $\varepsilon \bar{\Omega}_{p}$, where $\chi_{p}$ is constructed at the end of $\S 5$ and is a bounded viscosity solution of (5.14), (5.15).

We claim that for some $\rho>0, \varphi_{\varepsilon}$ is a viscosity supersolution of

$$
\lambda \varphi_{\varepsilon}(x)+H_{\varepsilon}\left(x, D \varphi_{\varepsilon}(x)\right) \geq 0 \quad x \in \varepsilon \bar{\Omega}_{p} \cap B_{\rho}(O),
$$

for all $\varepsilon$ such that $\varepsilon<\rho / R_{0}$.

Consider a smooth function $\zeta$ such that $\varphi_{\varepsilon}-\zeta$ attains its minimum in $\varepsilon \bar{\Omega}_{p} \cap B_{\rho}(O)$ at $\tilde{x}$ and $\varphi_{\varepsilon}(\tilde{x})=\zeta(\tilde{x})$. Then the function $z \mapsto \chi_{p}(z)+\psi_{p}(z)-\left(\psi_{p}(z)-\frac{1}{\varepsilon} \xi_{\varepsilon}(\varepsilon z)+\frac{1}{\varepsilon} \zeta(\varepsilon z)\right)$ attains its minimum at $\tilde{z}=\frac{\tilde{x}}{\varepsilon}$. From the definition of $\chi_{p}$ we get

$$
H_{0}\left(\tilde{z}, D \zeta(\tilde{x})-D \xi_{\varepsilon}(\tilde{x})+D_{z} \psi_{p}\left(\frac{\tilde{x}}{\varepsilon}\right)\right) \geq \bar{H}(O, p)
$$


and therefore,

$$
\lambda \varphi(O)+H_{0}\left(\tilde{z}, D \zeta(\tilde{x})-D \xi_{\varepsilon}(\tilde{x})+D_{z} \psi_{p}\left(\frac{\tilde{x}}{\varepsilon}\right)\right) \geq \lambda \varphi(O)+\bar{H}(O, p)=\gamma>0 .
$$

But

$$
\zeta(\tilde{x})-\varphi(O)=\xi_{\varepsilon}(\tilde{x})+\varepsilon \chi_{p}\left(\frac{\tilde{x}}{\varepsilon}\right) .
$$

From (6.5) and (6.6), it is possible to choose $\rho$ small enough such that

1. $\lambda \sup _{0<\varepsilon<\frac{\rho}{R_{0}}} \max _{x \in \varepsilon \bar{\Omega}_{p} \cap B_{\rho}(O)}\left|\xi_{\varepsilon}(x)\right| \leq \frac{\gamma}{6}$

2. $\lambda \sup _{0<\varepsilon<\frac{\rho}{R_{0}}} \max _{x \in \varepsilon \bar{\Omega}_{p} \cap B_{\rho}(O)}\left|\varepsilon \chi_{p}\left(\frac{x}{\varepsilon}\right)\right| \leq \frac{\gamma}{6}$

3. $\sup _{0<\varepsilon<\frac{\rho}{R_{0}}} \max _{x \in \varepsilon \bar{\Omega}_{p} \cap B_{\rho}(O)}\left|H_{0}\left(\frac{x}{\varepsilon}, D \zeta(x)-D \xi_{\varepsilon}(x)+D_{z} \psi_{p}\left(\frac{x}{\varepsilon}\right)\right)-H_{0}\left(\frac{x}{\varepsilon}, D \zeta(x)\right)\right| \leq \frac{\gamma}{6}$

We get that

$$
\lambda \zeta(\tilde{x})+H_{0}(\tilde{z}, D \zeta(\tilde{x})) \geq \frac{\gamma}{2} .
$$

Moreover, from the definition of $\ell_{\varepsilon}$, the continuity of the functions $\ell_{i}$ and the compactness of the set $[-1,1] \times A$, we see that

$$
\lim _{\rho \rightarrow 0} \sup _{0<\varepsilon<\frac{\rho}{R_{0}}}\left\|H_{0}\left(\frac{x}{\varepsilon}, p\right)-H_{\varepsilon}(x, p)\right\|_{L^{\infty}\left(\varepsilon \bar{\Omega}_{p} \cap B_{\rho}(O)\right)}=0 .
$$

Therefore, for $\rho$ small enough,

$$
\lambda \zeta(\tilde{x})+H_{\varepsilon}(\tilde{x}, D \zeta(\tilde{x})) \geq \frac{\gamma}{4}
$$

which proves the claim.

From (1.5) and (6.7), we deduce that

$$
\sup _{\varepsilon \bar{\Omega}_{p} \cap B_{\rho}(O)} u_{\varepsilon}-\varphi_{\varepsilon} \leq \sup _{\varepsilon \Omega_{p} \cap \partial B_{\rho}(O)} u_{\varepsilon}-\varphi_{\varepsilon} .
$$

Passing to the limit when $\varepsilon \rightarrow 0$, we obtain

$$
0=\bar{u}(O)-\varphi(O) \leq \bar{u}\left(\rho e_{i}\right)-\varphi\left(\rho e_{i}\right), \quad \forall i \in \mathcal{I}(p) .
$$

Since we can choose $\rho$ as small as we like, we reach a contradiction because $O$ is a strict maximum of $\bar{u}-\varphi$.

Second case: $\bar{H}(O, p) \leq E$. In this case (6.4) becomes:

$$
\lambda \varphi(O)+E=\gamma>0 .
$$

Let us modify $\varphi$ by taking $\tilde{\varphi}(x)=\varphi(O)+M|x|$. We choose $M$ large enough such that

- $M \geq q$ where $q$ is defined in Remark 3.2

- the function $\tilde{\varphi}$ is still an admissible test-function such that $\bar{u}-\tilde{\varphi}$ has a strict local maximum at $O$ and $\bar{H}(O, D \tilde{\varphi}(O)) \leq \bar{H}(O, p)$, so

$$
\max (E, \bar{H}(O, D \tilde{\varphi}(O))) \leq \max (E, \bar{H}(O, p))=E .
$$

Therefore, (6.4) holds for $\tilde{\varphi}$. 
For simplicity, we agree to write $\varphi=\tilde{\varphi}$.

Thanks to Remark 3.2, there exists $R^{*}$ such that the function $\tilde{w}^{R^{*}}(z)$ defined in (3.12) (recall that $M \geq q$ ) is a viscosity supersolution of

$$
H_{0}\left(z, D \tilde{w}^{R^{*}}(z)\right) \geq E-\frac{\gamma}{4} \quad \text { for any } z \in \bar{\Omega} .
$$

Note that, with $z^{R^{*}}$ defined in (3.12), if $z \in \bar{\Omega} \backslash \bar{\Omega}^{R^{*}}$ then $\tilde{w}^{R^{*}}(z)-\varphi(z)=\tilde{w}^{R^{*}}\left(z^{R^{*}}\right)-$ $\varphi(O)-M R^{*}$.

Let us call $\varphi_{\varepsilon}^{*}$ the function defined in $\varepsilon \bar{\Omega}$ by $\varphi_{\varepsilon}^{*}(x)=\varphi(O)+\varepsilon \tilde{w}^{R^{*}}\left(\frac{x}{\varepsilon}\right)$,

then if $\frac{x}{\varepsilon} \in \bar{\Omega} \backslash \bar{\Omega}^{R^{*}}$

$$
\varphi_{\varepsilon}^{*}(x)-\varphi(x)=\varepsilon\left(\tilde{w}^{R^{*}}\left(\frac{x}{\varepsilon}\right)-M \frac{|x|}{\varepsilon}\right)=O(\varepsilon) .
$$

For $\rho>0$ smaller than a constant independent of $\varepsilon$, we claim that $\varphi_{\varepsilon}^{*}$ is a viscosity supersolution of

$$
\lambda \varphi_{\varepsilon}^{*}(x)+H_{\varepsilon}\left(x, D \varphi_{\varepsilon}^{*}(x)\right) \geq O \quad x \in \varepsilon \bar{\Omega} \cap \overline{B_{\rho}(O)},
$$

for all $\varepsilon>0$. Let $\zeta$ a regular function and $x^{*} \in \varepsilon \bar{\Omega} \cap \overline{B_{\rho}(O)}$ be such that

$$
\varphi_{\varepsilon}^{*}(x)-\zeta(x) \geq \varphi_{\varepsilon}^{*}\left(x^{*}\right)-\zeta\left(x^{*}\right)=0, \quad x \in \varepsilon \bar{\Omega} \cap \overline{B_{\rho}(O)},
$$

This implies that, changing the variable $z=\frac{x}{\varepsilon}$ and $z^{*}=\frac{x^{*}}{\varepsilon}$

$$
\tilde{w}^{R^{*}}(z)-\frac{1}{\varepsilon} \zeta(\varepsilon z) \geq \tilde{w}^{R^{*}}\left(z^{*}\right)-\frac{1}{\varepsilon} \zeta\left(\varepsilon z^{*}\right)
$$

It is possible to use the equation satisfied by $w^{R^{*}}$ :

$$
\begin{gathered}
H_{0}\left(\frac{x^{*}}{\varepsilon}, D \zeta\left(x^{*}\right)\right) \geq E-\frac{\gamma}{4} . \\
\text { If } z^{*}=\frac{x^{*}}{\varepsilon} \in \bar{\Omega}^{R^{*}} \\
\lambda \varphi_{\varepsilon}^{*}\left(x^{*}\right)+H_{\varepsilon}\left(x^{*}, D \zeta\left(x^{*}\right)\right)=\lambda \varphi_{\varepsilon}^{*}\left(x^{*}\right)+H_{\varepsilon}\left(x^{*}, D \zeta\left(x^{*}\right)\right)-H_{0}\left(\frac{x^{*}}{\varepsilon}, D \zeta\left(x^{*}\right)\right)+H_{0}\left(\frac{x^{*}}{\varepsilon}, D \zeta\left(x^{*}\right)\right) \\
\geq \lambda \varphi(0)-C\left(R^{*}\right) \varepsilon+H_{\varepsilon}\left(x^{*}, D \zeta\left(x^{*}\right)\right)-H_{0}\left(\frac{x^{*}}{\varepsilon}, D \zeta\left(x^{*}\right)\right)+H_{0}\left(\frac{x^{*}}{\varepsilon}, D \zeta\left(x^{*}\right)\right) \\
\geq-E+\gamma+\left(E-\frac{\gamma}{4}\right)-C\left(R^{*}\right) \varepsilon+H_{\varepsilon}\left(x^{*}, D \zeta\left(x^{*}\right)\right)-H_{0}\left(\frac{x^{*}}{\varepsilon}, D \zeta\left(x^{*}\right)\right) \\
=\frac{3}{4} \gamma+O(\varepsilon)+H_{\varepsilon}\left(x^{*}, D \zeta\left(x^{*}\right)\right)-H_{0}\left(\frac{x^{*}}{\varepsilon}, D \zeta\left(x^{*}\right)\right) \\
=\frac{1}{2} \gamma+H_{\varepsilon}\left(x^{*}, D \zeta\left(x^{*}\right)\right)-H_{0}\left(\frac{x^{*}}{\varepsilon}, D \zeta\left(x^{*}\right)\right)
\end{gathered}
$$

If $z^{*}=\frac{x^{*}}{\varepsilon} \in \bar{\Omega} \backslash \bar{\Omega}^{R^{*}}$

$$
\lambda \varphi_{\varepsilon}^{*}\left(x^{*}\right)+H_{\varepsilon}\left(x^{*}, D \zeta\left(x^{*}\right)\right) \geq-C \rho+\frac{1}{2} \gamma+H_{\varepsilon}\left(x^{*}, D \zeta\left(x^{*}\right)\right)-H_{0}\left(\frac{x^{*}}{\varepsilon}, D \zeta\left(x^{*}\right)\right)
$$

Using an argument similar to that used to prove (6.8) for $\rho$ small enough we can deduce from (6.9) and (6.10)

$$
\lambda \varphi_{\varepsilon}^{*}\left(x^{*}\right)+H_{\varepsilon}\left(x^{*}, D \zeta\left(x^{*}\right)\right) \geq 0 .
$$

We deduce from this that

$$
\sup _{\varepsilon \bar{\Omega} \cap B_{\rho}(O)} u_{\varepsilon}-\varphi_{\varepsilon}^{*} \leq \sup _{\varepsilon \Omega \cap \partial B_{\rho}(O)} u_{\varepsilon}-\varphi_{\varepsilon}^{*}
$$

which leads to a contradiction as above. 
$\underline{u}$ is a supersolution of (6.1) We are left with proving that $\underline{u}$ is a supersolution of (6.1). We consider a strict minimum $\bar{x}$ of $\underline{u}-\tilde{\varphi}$, such that $\underline{u}(\bar{x})=\tilde{\varphi}(\bar{x})$, where $\tilde{\varphi}$ is a regular test-function for (6.2)-(6.1). In the case when $\bar{x} \neq O$, the desired result is proved in [3]. Thus, we may focus on the case when $\bar{x}=O$.

We want to show that

$$
\lambda \tilde{\varphi}(O)+\max (E, \bar{H}(O, D \tilde{\varphi}(O))) \geq 0 .
$$

We suppose by contradiction that

$$
\lambda \tilde{\varphi}(O)+\max (E, \bar{H}(O, D \tilde{\varphi}(O)))=\tilde{\gamma}<0 .
$$

Let $\tilde{p} \in \mathbb{R}_{N}$ be defined by $\tilde{p}=\left(D \tilde{\varphi}\left(O, e_{1}\right), \ldots, D \tilde{\varphi}\left(O, e_{N}\right)\right)$

First case If $\bar{H}(O, \tilde{p})>E$ and $\mathcal{I}(\tilde{p})=\{1, \ldots, N\}$, i.e. for all $i \in\{1, \ldots, N\}, \bar{H}_{i}^{+}\left(O, D \tilde{\varphi}\left(0, e_{i}\right)\right)=$ $\bar{H}(O, \tilde{p})$, then we choose $\varphi=\tilde{\varphi}$, and $p=\tilde{p}$.

Second case On the contrary, i.e. if either $\bar{H}(O, \tilde{p}) \leq E$ or $\bar{H}(O, \tilde{p})>E$ and $\mathcal{I}(\tilde{p}) \neq$ $\{1, \ldots, N\}$, it is possible to add a piecewise linear nonpositive function vanishing at $O$ to $\tilde{\varphi}$ and obtain a new function $\varphi$ such that

- $O$ is a strict minimum of $\underline{u}-\varphi$, and $\underline{u}(O)=\varphi(O)$

- if $p=\left(D \varphi\left(O, e_{1}\right), \ldots, D \varphi\left(O, e_{N}\right)\right)$, then $\lambda \varphi(O)+\bar{H}(O, p)=\gamma<0$

- $\bar{H}(O, p)>E$ and $\mathcal{I}(p)=\{1, \ldots, N\}$, i.e. for all $i \in\{1, \ldots, N\}, \bar{H}_{i}^{+}\left(O, p_{i}\right)=\bar{H}(O, p)$.

We then consider $\psi_{p}$ as in (5.1), $\chi_{p}$ defined at the end of $\S 5$ with $\bar{\Omega}_{p}=\bar{\Omega}$, and $\xi_{\varepsilon}$ as above. The perturbed test function is defined in $\varepsilon \Omega$ by

$$
\varphi_{\varepsilon}(x)=\varphi(O)+\xi_{\varepsilon}(x)+\varepsilon \chi_{p}\left(\frac{x}{\varepsilon}\right) .
$$

We claim that for some $\rho>0, \varphi_{\varepsilon}$ is a viscosity subsolution of

$$
\lambda \varphi_{\varepsilon}(x)+H_{\varepsilon}\left(x, D \varphi_{\varepsilon}(x)\right) \leq 0 \quad x \in \varepsilon \Omega \cap B_{\rho}(O),
$$

for all $\varepsilon$ such that $R_{0} \varepsilon<\rho$. The proof of the claim is essentially the same as for (6.7).

The comparison principle implies that

$$
\inf _{\varepsilon \bar{\Omega} \cap B_{\rho}(O)} u_{\varepsilon}-\varphi_{\varepsilon} \geq \inf _{\varepsilon \Omega \cap \partial B_{\rho}(O)} u_{\varepsilon}-\varphi_{\varepsilon} .
$$

and then

$$
0=\underline{u}(O)-\varphi(O) \geq \inf _{\mathcal{G} \cap \partial B_{\rho}(O)} \underline{u}-\varphi .
$$

Since we can choose $\rho$ as small as we like, we reach a contradiction because $O$ is a strict minimum of $\underline{u}-\varphi$.

Proof of Theorem 4.2. From Theorem 6.1, the relaxed semilimits $\bar{u}$ and $\underline{u}$ are respectively bounded viscosity subsolution and supersolution of (4.10)-(4.11). By comparison (Theorem 4.1), this implies that $\bar{u}=\underline{u}=u$ and the local uniform convergence of $u_{\varepsilon}$ to $u$. 


\section{A Proofs of Propositions 2.1 and 2.2}

Proof of Proposition 2.1 Let us prove that Assumption 2.1 holds with $L_{i}=0$.

Consider first the case when $p_{i}<\underline{p}_{i}$ : from the convexity assumptions, there exists $\bar{a} \in A$ and $\bar{y} \in[-1,1]$ such that $\bar{a}_{i}^{\perp}=0$ and

$$
\bar{H}_{i}\left(0, p_{i}\right)=-p_{i} \bar{a}_{i}-\ell_{i}(0, \bar{y}, \bar{a}) .
$$

Since $\bar{H}_{i}\left(0, p_{i}\right)>\Lambda_{i}^{0}$, we know that $\bar{a}_{i} \geq 0$. With the constant $r$ appearing in Assumption 1.1, if $\tilde{t}=\frac{\left|\bar{y}-y_{0}\right|}{r}$, the control law $\tilde{\alpha}$ defined by

$$
\begin{aligned}
\tilde{\alpha}(s)=r \operatorname{sign}\left(\bar{y}-y_{0}\right) e_{i}^{\perp} & \text { if } 0<s<\tilde{t}, \\
\tilde{\alpha}(s)=\bar{a} & \text { if } \tilde{t}<s,
\end{aligned}
$$

belongs to $\mathcal{A}_{i, y_{0}}$. Moreover $\int_{0}^{t} \tilde{\alpha}_{i}(s) d s=\bar{a}_{i}(t-\tilde{t})_{+} \geq 0$. It is clear that if $t \geq 2 / r$, then $t \geq \tilde{t}$ and

$$
\begin{aligned}
\int_{0}^{t}\left(p_{i} \tilde{\alpha}_{i}(s)+\ell_{i}(0, \tilde{y}(s), \tilde{\alpha}(s))\right) d s & \leq-(t-\tilde{t}) \bar{H}_{i}\left(0, p_{i}\right)+\frac{2}{r} \max _{(y, a) \in[-1,1] \times A} \ell_{i}(0, y, a) \\
& \leq-t \bar{H}_{i}\left(0, p_{i}\right)+c,
\end{aligned}
$$

for a constant $c$ possibly depending on $p_{i}$ and $\tilde{y}(t)=y_{0}+\int_{0}^{t} \tilde{\alpha}_{i}^{\perp}(s) d s$. With $C$ as in (2.9), we deduce that

$$
\int_{0}^{t}\left(p_{i} \tilde{\alpha}_{i}(s)+\ell_{i}(0, \tilde{y}(s), \tilde{\alpha}(s))\right) d s \leq v_{i}\left(0, p_{i}, y_{0}, t\right)+C+c .
$$

It is always possible to choose $C_{i} \geq C+c$ such that for all $0 \leq t \leq 2 / r$, there is an admissible control law such that $\int_{0}^{s} \tilde{\alpha}_{i}(\tau) d \tau \geq 0, \quad \forall 0 \leq s \leq t$ and (2.19) holds.

We are left with the case when $p_{i} \in \operatorname{argmin} \bar{H}_{i}(0, \cdot)$ : in that case, there exists $\bar{y} \in[-1,1]$ such that

$$
\bar{H}_{i}\left(0, p_{i}\right)=-\ell_{i}(0, \bar{y}, 0) .
$$

We can repeat the arguments above with $\bar{a}=0$ and prove the claim.

Proof of Proposition 2.2 For brevity, we only discuss the case when $p_{i}<\underline{p}_{i}$, because the case $p_{i} \in\left[\underline{p}_{i}, \bar{p}_{i}\right]$ is handled in the same manner.

By adding the constant $\Lambda_{i}^{0}$ to $\ell_{i}$, it is always possible to assume that $\Lambda_{i}^{0}=0$ and

$$
\underline{c}_{i}|a|^{\nu_{i}} \leq \ell_{i}(0, y, a) \leq \bar{c}_{i}|a|^{\nu_{i}} .
$$

Thus, $0 \in\left[\underline{p}_{i}, \bar{p}_{i}\right]$.

Step 1 Consider $y_{0} \in[-1,1]$, and $(\alpha, T) \in \mathcal{A}_{i, y_{0}} \times \mathbb{R}_{+}$such that $\min _{0 \leq \theta \leq T} \int_{0}^{\theta} \alpha_{i}(s) d s \leq-L_{i}$. Let $\theta$ be such that $\int_{0}^{\theta} \alpha_{i}(s) d s \leq-L_{i}$. Hölder's inequality yields

$$
L_{i} \leq \int_{0}^{\theta} \alpha_{i}^{-}(s) d s \leq \theta^{1-\frac{1}{\nu_{i}}}\left(\int_{0}^{\theta}|\alpha(s)|^{\nu_{i}} d s\right)^{\frac{1}{\nu_{i}}},
$$

thus

$$
\int_{0}^{\theta}|\alpha(s)|^{\nu_{i}} d s \geq L_{i}^{\nu_{i}} \theta^{1-\nu_{i}}
$$


Hence, any $\alpha$ such that

$$
\begin{array}{r}
\int_{0}^{T} \alpha_{i}(s) d s=0, \\
\min _{0 \leq \theta \leq T} \int_{0}^{\theta} \alpha_{i}(s) d s \leq-L_{i}
\end{array}
$$

satisfies

$$
\int_{0}^{T} \ell_{i}(0, y(s), \alpha(s)) d s \geq \underline{c}_{i} L_{i}^{\nu_{i}} T^{1-\nu_{i}}
$$

with

$$
y(s)=y_{0}+\int_{0}^{s} \alpha_{i}^{\perp}(\tau) d \tau .
$$

Moreover, if $L_{i}>\frac{2 \max _{A} a_{i}^{-}}{r}$, where $r$ is the constant in Assumption 1.1, then (A.3) implies that $T>\frac{2}{r}$. Therefore, from Assumption 1.1, the control

$$
\bar{\alpha}=\bar{\alpha}_{i}^{\perp} e_{i}^{\perp}=\frac{y(T)-y_{0}}{T} e_{i}^{\perp}=\frac{1}{T} \int_{0}^{T} \alpha(\tau) d \tau
$$

belongs to $A$.

On the other hand, with $\bar{\alpha}$ defined by (A.5), (2.23) implies that

$$
\int_{0}^{T} \ell_{i}\left(0, y_{0}+\bar{\alpha}_{i}^{\perp} s, \bar{\alpha}\right) d s \leq \bar{c}_{i} 2^{\nu_{i}} T^{1-\nu_{i}} .
$$

Hence, from (A.4) and (A.6), it is possible to choose $L_{i}$ large enough, namely larger than $\max \left(\frac{2 \max _{A} a_{i}^{-}}{r}, 2\left(\frac{\bar{c}_{i}}{\underline{c}_{i}}\right)^{\frac{1}{\nu_{i}}}\right)$, such that for all $(T, \alpha)$ satisfying (A.2) (A.3), $\bar{\alpha}$ defined by (A.5) is an admissible control in $[0, T]$ and the following inequality holds

$$
\int_{0}^{T} \ell_{i}(0, y(s), \alpha(s)) d s \geq \int_{0}^{T} \ell_{i}\left(0, y_{0}+\bar{\alpha}_{i}^{\perp} s, \bar{\alpha}\right) d s .
$$

Step 2 Take $L_{i}$ as in the conclusion of Step 1 and $p_{i}$ such that $p_{i}<\underline{p}_{i}$. Therefore $p_{i}<0$. Since $\Lambda_{i}^{0}=0$, we also have that $\bar{H}_{i}\left(0, p_{i}\right)>0$. Take $C$ as in $(2.9)$ : for $t>\frac{2 C}{\bar{H}_{i}\left(0, p_{i}\right)}, v_{i}\left(0, p_{i}, y_{0}, t\right)<-C$. There exists a control law $\alpha \in \mathcal{A}_{i, y_{0}}$ such that

$$
\int_{0}^{t}\left(p_{i} \alpha_{i}(s)+\ell_{i}(0, y(s), \alpha(s))\right) d s \leq v_{i}\left(0, p_{i}, y_{0}, t\right)+C<0
$$

where $y$ is given by (2.1). Then, $\int_{0}^{t} \alpha_{i}(s) d s>0$. If $\min _{0 \leq s \leq t} \int_{0}^{s} \alpha_{i}(\tau) d \tau \leq-L_{i}$, we see from Step 1 that it is possible to replace $\alpha$ by a control $\tilde{\alpha}$ satisfying (2.18) and such that

$$
\int_{0}^{t}\left(p_{i} \tilde{\alpha}_{i}(s)+\ell_{i}(0, \tilde{y}(s), \tilde{\alpha}(s))\right) d s \leq \int_{0}^{t}\left(p_{i} \alpha_{i}(s)+\ell_{i}(0, y(s), \alpha(s))\right) d s,
$$

hence (2.19) with $C_{i}=C$, and $\tilde{y}(t)=y_{0}+\int_{0}^{t} \tilde{\alpha}_{i}^{\perp}(s) d s$.

It is always possible to choose $C_{i} \geq C$ such that for all $t \leq \frac{2 C}{\bar{H}_{i}\left(0, p_{i}\right)}$, there exists an admissible control law such that (2.18) and (2.19) hold. 
Acknowledgement The authors would like to warmly thank Cyril Imbert, Régis Monneau and Antonio Siconolfi for very constructive discussions. The authors were partially funded by the ANR project ANR-12-BS01-0008-01. The first author was partially funded by the ANR project ANR-12-MONU-0013.

\section{References}

[1] Y. Achdou, F. Camilli, A. Cutrì, and N. Tchou, Hamilton-Jacobi equations constrained on networks, NoDEA Nonlinear Differential Equations Appl. 20 (2013), no. 3, 413-445. MR 3057137

[2] Y. Achdou, S. Oudet, and N. Tchou, Hamilton-Jacobi equations for optimal control on junctions and networks, 2013.

[3] O. Alvarez and M. Bardi, Viscosity solutions methods for singular perturbations in deterministic and stochastic control, SIAM J. Control Optim. 40 (2001/02), no. 4, 1159-1188 (electronic). MR 1882729 (2003a:49034)

[4] Singular perturbations of nonlinear degenerate parabolic PDEs: a general convergence result, Arch. Ration. Mech. Anal. 170 (2003), no. 1, 17-61. MR 2012646 (2004h:35012)

[5] O. Alvarez, M. Bardi, and C. Marchi, Multiscale problems and homogenization for second-order Hamilton-Jacobi equations, J. Differential Equations 243 (2007), no. 2, 349-387. MR 2371792 (2009a:35030)

[6] _ Multiscale singular perturbations and homogenization of optimal control problems, Geometric control and nonsmooth analysis, Ser. Adv. Math. Appl. Sci., vol. 76, World Sci. Publ., Hackensack, NJ, 2008, pp. 1-27. MR 2487745 (2010b:49024)

[7] Z. Artstein and V. Gaitsgory, The value function of singularly perturbed control systems, Appl. Math. Optim. 41 (2000), no. 3, 425-445. MR 1739395 (2001b:49035)

[8] F. Bagagiolo and M. Bardi, Singular perturbation of a finite horizon problem with state-space constraints, SIAM J. Control Optim. 36 (1998), no. 6, 2040-2060 (electronic). MR 1638940 (99h:49040)

[9] M. Bardi and I. Capuzzo-Dolcetta, Optimal control and viscosity solutions of Hamilton-JacobiBellman equations, Systems \& Control: Foundations \& Applications, Birkhäuser Boston Inc., Boston, MA, 1997, With appendices by Maurizio Falcone and Pierpaolo Soravia. MR 1484411 (99e:49001)

[10] G. Barles, Discontinuous viscosity solutions of first-order Hamilton-Jacobi equations: a guided visit, Nonlinear Anal. 20 (1993), no. 9, 1123-1134. MR 1216503 (94d:49047)

[11] G. Barles, A. Briani, and E. Chasseigne, A Bellman approach for regional optimal control problems in $R^{N}$, hal preprint hal:00825778 (2013).

$[12] \_$, A Bellman approach for two-domains optimal control problems in $\mathbb{R}^{N}$, ESAIM Control Optim. Calc. Var. 19 (2013), no. 3, 710-739. MR 3092359

[13] E. N. Barron and R. Jensen, Semicontinuous viscosity solutions for Hamilton-Jacobi equations with convex Hamiltonians, Comm. Partial Differential Equations 15 (1990), no. 12, 1713-1742. MR 1080619 (91h:35069)

[14] A. Bensoussan, Perturbation methods in optimal control, Wiley/Gauthier-Villars Series in Modern Applied Mathematics, John Wiley \& Sons Ltd., Chichester, 1988, Translated from the French by C. Tomson. MR 949208 (89m:93002)

[15] F. Camilli and C. Marchi, A comparison among various notions of viscosity solutions for HamiltonJacobi equations on network, preprint (2013).

[16] K.-J. Engel, M. Kramar Fijavž, R. Nagel, and E. Sikolya, Vertex control of flows in networks, Netw. Heterog. Media 3 (2008), no. 4, 709-722. MR MR2448938 (2009h:93011) 
[17] L. C. Evans, The perturbed test function method for viscosity solutions of nonlinear PDE, Proc. Roy. Soc. Edinburgh Sect. A 111 (1989), no. 3-4, 359-375. MR 1007533 (91c:35017)

[18] V. Gaitsgory and A. Leizarowitz, Limit occupational measures set for a control system and averaging of singularly perturbed control systems, J. Math. Anal. Appl. 233 (1999), no. 2, 461-475. MR 1689645 (2000c:93064)

[19] M. Garavello and B. Piccoli, Traffic flow on networks, AIMS Series on Applied Mathematics, vol. 1, American Institute of Mathematical Sciences (AIMS), Springfield, MO, 2006, Conservation laws models. MR MR2328174 (2008g:90023)

[20] C. Imbert and R. Monneau, The vertex test function for Hamilton-Jacobi equations on networks, arXiv preprint arXiv:1306.2428 (2013).

[21] C. Imbert, R. Monneau, and H. Zidani, A Hamilton-Jacobi approach to junction problems and application to traffic flows, ESAIM Control Optim. Calc. Var. 19 (2013), no. 1, 129-166. MR 3023064

[22] H. Ishii, A short introduction to viscosity solutions and the large time behavior of solutions of Hamilton-Jacobi equations, Hamilton-Jacobi Equations: Approximations, Numerical Analysis and Applications, Springer, 2013, pp. 111-249.

[23] P-L. Lions, Cours du Collège de France, http://www.college-de-france.fr/default/EN/all/equ_der/, january and february 2014.

[24] P-L. Lions, G. Papanicolaou, and S.R.S Varadhan, Homogenization of Hamilton-Jacobi equations, unpublished.

[25] D. Schieborn and F. Camilli, Viscosity solutions of Eikonal equations on topological networks, Calc. Var. Partial Differential Equations 46 (2013), no. 3-4, 671-686. MR 3018167

[26] G. Terrone, Limiting relaxed controls and averaging of singularly perturbed deterministic control systems, Dyn. Contin. Discrete Impuls. Syst. Ser. A Math. Anal. 18 (2011), no. 5, 653-672. MR 2884756

[27] M. Valadier, Sous-différentiels d'une borne supérieure et d'une somme continue de fonctions convexes, C. R. Acad. Sci. Paris Sér. A-B 268 (1969), A39-A42. MR 0241975 (39 \#3310) 